\title{
Nonlinear dynamics of large-scale coherent structures in turbulent free shear layers
}

\author{
Xuesong $\mathrm{Wu}^{1,2} \quad$ Xiuling Zhuang ${ }^{1}$ \\ ${ }^{1}$ Department of Mechanics, Tianjin University, P. R. China \\ ${ }^{2}$ Department of Mathematics, Imperial College London, \\ 180 Queen's Gate, London SW7 2AZ, UK
}

October 22, 2015

\begin{abstract}
Fully developed turbulent free shear layers exhibit a high degree of order, characterized by large-scale coherent structures in the form of spanwise vortex rollers. Extensive experimental investigations show that such organised motions bear remarkable resemblance to instability waves, and their main characteristics, including the length scales, propagation speeds and transverse structures, are reasonably well predicted by linear stability analysis of the mean flow. In this paper, we present a mathematical theory to describe the nonlinear dynamics of coherent structures. The formulation is based on the triple decomposition of the instantaneous flow into a mean field, coherent fluctuations and small-scale turbulence but with the mean-flow distortion induced by nonlinear interactions of coherent fluctuations being treated as part of the organised motion. The system is closed by employing gradient type of models for the time- and phase-averaged Reynolds stresses of fine-scale turbulence. In the high-Reynolds-number limit, the nonlinear non-equilibrium critical-layer theory for laminar-flow instabilities is adapted to turbulent shear layers by accounting for (a) the enhanced non-parallelism associated with fast spreading of the mean flow, and (b) the influence of small-scale turbulence on coherent structures. The combination of these factors with nonlinearity leads to an interesting evolution system, consisting of the coupled amplitude and vorticity equations, in which non-parallelism contributes the so-called translating critical-layer effect. Numerical solutions of the evolution system capture vortex roll-up, which is the hallmark of turbulent mixing layer, and the predicted amplitude development mimics the qualitative feature of oscillatory saturation that has been observed in a number of experiments. A fair degree of quantitative agreement is obtained with one set of experimental data.
\end{abstract}

\section{Introduction}

While turbulence generally refers to spatially and temporally random and chaotic fluid motions, it has been widely recognized and accepted since the 1970s that orderly and quasi-deterministic fluctuations, referred to as coherent structures (CS), are also present in turbulent shear flows. A precise and universally accepted definition of coherent structures is not presently available and indeed may not ever be possible. The consensus is that they represent the portion of a fluid motion which is vortical and correlated over fairly long length and time scales. The statistical correlations are likely to be underpinned by fluctuations exhibiting relatively regular spatial patterns and quasi-periodic reoccurrence. Since the latter are masked by random small-scale fluctuations, CS could only be reliably 
detected by careful flow visualization (e.g. Brown \& Roshko 1974) and elaborated phase or ensemble average (e.g. Hussain \& Zaman 1985). By using these techniques, much information has been gained about properties and dynamical significance of CS. The main findings have been comprehensively reviewed by Cantel (1981), Hussain (1983) and Wygnanski \& Petersen (1987) among others. CS are known to appear in boundary layers as well as in free shear layers. Our interest will be on the latter, which include mixing layers, wake and (planar or axisymmetric) jets.

Brown \& Roshko (1974) provided the first visualizations of CS in a high-Reynoldsnumber turbulent mixing layer. The striking images, which were to become iconic, showed clearly that CS were predominantly two dimensional, consisting of an array of spanwise concentrated vortices. These vortices all propagate at a constant speed, which is approximately the average of the free-stream velocities. Their length scale is comparable with the local shear-layer thickness. Winant \& Browand (1974) observed that adjacent vortices appear to merge repeatedly to form larger structures, a process referred to as 'vortex pairing'. Further visualization and measurements reaffirmed that CS are quasitwo-dimensional, persist despite strong external turbulence (Wygnanski et al. 1979) and reside in the asymptotic self-preserving region far downstream (Browand \& Troutt 1980). These observations led to the viewpoint that the instantaneous flow field can be decomposed into a mean field, CS and small-scale turbulence (Reynolds \& Hussain 1972).

CS were observed in plane jets (Hussaini \& Thompson 1980, Antonia et al. 1983) and in the far field of a wake (e.g. Wygnanski, Champagne \& Marasli 1986). These flows have symmetric mean profiles, and hence CS consist of two arrays of counter-rotating spanwise vortices. Observation of CS in a circular jet (Crow \& Champagne 1971) preceded those in plane mixing layers. In the region near the nozzle, vortex rings are the dominant CS, taking the place of spanwise vortex rollers, but helical structures appear as well. As the shear layer thickens with the downstream distance, vortex rings attenuate while helical structures become more significant.

Experimental studies indicate that CS arise whether the upstream boundary layer (over the splitter plate or in the nozzle) is laminar or turbulent, tripped or non-tripped. On the other hand, the mean-flow properties and fluctuations do exhibit sensitive dependence on upstream conditions including the disturbance level, the state and thickness of the upstream boundary layer (Batt 1975, Weisbrot, Einav \& Wygnanski 1982), and the thickness of the splitter plate or nozzle (Dziomba \& Fiedler 1985). Remarkably, the impact may persist over a very long distance before the self-preserving regime is reached, and furthermore even when the mean flow has already acquired a self-similarity shape, the imprint of initial conditions may remain in the shear-layer thickness.

Many experiments have been conducted in which time-periodic disturbances are introduced in a controlled manner. The resulting CS appear more organized so that their characteristics can be studied in greater detail than otherwise possible. Using a small flap at the trailing edge of the splitter plate, Oster \& Wygnanski (1982) introduced into a mixing layer a disturbance with a frequency an order-of-magnitude smaller than the characteristic frequency of the initial shear layer. At small amplitudes, the spreading rate is enhanced. At larger amplitudes, the forcing resonates with the mixing layer in a streamwise region, where the spreading rate is suppressed, and most significantly the energy is extracted from turbulence and transferred into the mean flow.

Gaster, Kit \& Wygnanski (1985, hereafter referred to as GKW) focussed on smallamplitude disturbances, excited using the same method as in Oster \& Wygnanski (1982). The characteristic wavelength, propagation speed and transverse distribution of CS were measured and found to be fairly well predicted by the linear stability analysis of the mean flow. Fiedler \& Mensing (1985) investigated a one-stream shear layer forced by periodic 
disturbances of various frequency and amplitude. Compared with the 'neutral' (i.e. unforced) case, vortex rollers are more sharply defined. They amplify as they propagate downstream, and reach a saturation point $x_{s}$ before decaying. It was found that $x_{s}$ is inversely proportional to the frequency, consistent with linear stability theory. The amplitude development of the periodic signal was mapped out. For weak excitations, the evolution of CS of different frequency follows a universal rule provided that the amplitude and distance are suitably normalized. For strong excitations, the attenuation may be oscillatory. Weisbrot \& Wygnanski (1988, hereafter referred to as WW) investigated CS in a shear layer subject to high-amplitude excitation. They measured both the streamwise and the transverse phase-averaged velocities, from which the vorticity and Reynolds stress were determined. The transverse distributions of the velocities and Reynolds stress were found to be well predicted by the linear stability analysis of the time averaged mean flow despite high intensity of the disturbance. However, the amplification rate of CS was poorly predicted. Wygnanski \& Weisbrot (1988) investigated the vortex pairing process in detail by introducing both fundamental and subharmonic disturbances. They found that roll-up and pairing may both be attributed to redistribution of vorticity taking place when the fundamental and subharmonic modes approach their respective neutral positions (as predicted by linear inviscid stability analysis).

Wygnanski et al. (1986) measured the phase-averaged velocity of forced sinuous disturbances in a small-deficit turbulent wake, while varicose modes were investigated subsequently by Marasli, Champagne \& Wygnanski (1989). Linear non-parallel stability theory predicts rather well the shapes of both modes as well as the growth rate of the sinuous mode, but not that of the varicose mode. Marasli, Champagne \& Wygnanski $(1991,1992)$ measured the coherent Reynolds stress, which is generated by nonlinear interactions of CS. The calculation using the eigenfunction of linear stability analysis captures the transverse distribution of the Reynolds stress in the region upstream of the neutral position, approaching which nonlinearity becomes significant. Under high-amplitude excitations, the Reynolds stress was found to change its sign in the neighbourhood of the neutral position. Hussain \& Thompson (1980) investigated CS in the near field of a plane jet. As in the plane mixing layer, the transverse distribution of the velocity is in agreement with the prediction of linear stability theory. However, the phase distribution is not.

CS in circular jets have received much attention since the pioneering work of Crow \& Champagne (1971). Zaman \& Hussain (1980) and Hussain \& Zaman (1981) investigated vortex pairing process subject to excitation. Extensive measurements of fundamental, harmonic and (in the case of pairing) subharmonic components as well as the Reynolds stress were carried out. However, the results were not interpreted in the framework of hydrodynamic instability. More recently, Suzuki \& Colonius (2006) detected axisymmetric and first two helical modes in a subsonic circular jet, and mapped out their development from the nozzle to the end of the potential core, while Oberleithner, Rukes \& Soria (2014) focussed on axisymmetric modes. Linear stability calculations were performed, and the agreement with measurements supports the notation that CS behave, in a statistical sense, as instability waves on the turbulent mean flow. It is worth noting that the calculations of Oberleithner et al. (2014) were carried out for different levels of excitation, and in each case the mean-flow profile used is that specifically measured for the given excitation.

While two-dimensional CS (of the kind as observed in the experiment of Brown \& Roshko (1974)) have received much emphasis and attention, three-dimensional CS have also been observed and studied. The reader is referred to Nygaard \& Glezer (1994) and Estevadeordal \& Kleis (2002) as well as the references therein for further details.

The discovery of CS has profoundly changed our perception of turbulence (Roshko 1976), and their potential dynamical significance could be immense for both modelling 
and practical control of turbulence. It is well known that the gradient type of closure models lacks universality. The failure was attributed to the fact that large-scale CS are fundamentally influenced by upstream and boundary conditions, and the Reynolds stresses generated by them cannot be characterised by the local strain rate of the mean flow and an eddy viscosity. For the same reason, scalar transport and mixing models of the usual gradient type also fail. A possible strategy for improved modelling is to decompose fluctuations into large and small scales. The latter are expected to be controlled by local dynamics so that a gradient type of model for their contribution to Reynolds stresses might suffice. Now if the large scales could be treated separately with the role of boundary and upstream conditions being taken into account, there may be a prospect of developing closure models that are more physics-based and thus have wider validity. Experimental studies in the past 40 years acquired abundant evidence and data supporting the dynamical role of CS. CS control entrainment and mixing of species (Dimotakis \& Brown 1976, Dutton \& Lucht 2006), and contribute comparable amount to the Reynolds stresses and heat flux as does random turbulence (Hussain \& Zaman 1985, Antonia et al. 1986). The Reynolds stresses may take negative values in the region of positive mean-flow gradient, indicating that closure models of the mean-flow gradient type are inappropriate.

It has long been suggested that CS in turbulent jets, like instability modes in transitional stage, might be dominant sources of noise (Bishop, Ffowcs Williams \& Smith 1971, Crow \& Champagne 1971). Experiments show that CS are instrumental in generating noise. For instance, excitation of CS by pure-tone forcing on a jet could amplify broadband noise if the frequency is relatively low (Bechert \& Pfizenmaier 1975), or suppress the noise if the frequency is high (Hussain \& Hasan 1985). More direct evidence that CS may emit noise was provided recently by Cavalieri et al. (2013) and Suzuki (2013). Latest efforts of modelling noise generation by CS have been reviewed by Jordan \& Colonius (2013), but the precise mechanisms remain to be understood fully. They are probably similar to the mechanisms by which instability modes in a laminar jet radiate sound; those mechanisms have been described on the basis of first principles by Tam \& Burton (1984) and $\mathrm{Wu}(2005)$ for supersonic and by Wu \& Huerre (2009) for subsonic regimes.

In order to develop appropriate physics-based models for Reynolds stresses, mixing (entrainment) and noise generation, it is necessary to acquire better physical understanding and quantitative descriptions of CS. As has been indicated above, linear stability theory of the mean flow predicts the shape, propagation speed and characteristic length scale of CS reasonably well. The concept of instability appears to offer a viable theoretical framework for describing the dynamics of CS despite objections by some investigators (see e.g. Hussain 1983). The problem of poor prediction of amplification rates has been alleviated by accounting for non-parallelism by using the approach of Crighton \& Gaster (1975) as was done by GKW and Wygnanski et al. (1986). More recently, linear parabolized-stabilityequation (PSE) approach was applied to study the evolution of CS (Gudmundsson \& Colonius 2011), leading to improved accuracy in certain range of parameters.

The theoretical studies mentioned above ignored nonlinearity and small-scale turbulence completely. The development of CS under the influence of these effects was tackled by Liu \& Merkine (1976) and Alper \& Liu (1978), who adapted the energy method based on the so-called 'shape assumptions': (a) the mean flow assumes a given self-similar shape but with its thickness evolving; (b) CS retains the shape of the eigenfunction of the linear stability problem; (c) the mean Reynolds stresses take a presumed shape, while the phase-averaged Reynolds stresses, which account for the effect of fine-scale turbulence on CS, were modelled with the aid of the transport equations. From the transversely integrated kinetic energy equations for the mean flow, CS and small-scale turbulence, a coupled system governing the shear-layer thickness, the amplitude of CS and the turbu- 
lence energy, was derived. The model involves a fair degree of empiricism, but captures essential physics, and indeed numerical solutions appeared to predict some of experimental observations qualitatively (Mankbadi \& Liu 1981, Liu 1989). Cohen, Marasli \& Levinski (1994) considered interaction between CS and the mean flow. At each location, the CS is treated as an instability mode of the mean flow. The local eigenfunction and eigenvalue are used to evaluate the Reynolds stresses, which are then used to calculate a corrected mean flow downstream, where the above procedure is repeated. The approach, which ignores harmonics as well as small-scale turbulence, was found to predict fairly well the spreading rate of the shear-layer thickness up to the neutral position, in the vicinity of which the theory fails. While nonlinear theories above have achieved some success, unfortunately none of them was able to predict vortex roll-up.

Nonlinear development of instability modes in transitional laminar shear flows has been extensively studied, where in order to account for systematically competing physical factors (such as nonlinearity, viscosity, non-parallelism and non-equilibrium), a high-Reynoldsnumber asymptotic approach has been adopted. This has led to nonlinear critical-layer theory; for reviews see Goldstein (1995) and Cowley \& Wu (1994). This approach was based on the key understanding that as an initially small instability mode propagates downstream, its growth rate decreases due to the gradual thickening of the shear layer and a critical layer emerges at the transverse location where the base-flow velocity equals the phase speed of the mode. The disturbance then enters a nonlinear evolution stage due to enhanced nonlinear effects within the critical layer. For a two-dimensional mode on a base flow with an inflectional profile, the critical layer dynamics turns out to be strongly nonlinear. As a result, the vorticity of the disturbance rolls up to form Kelvin-Helmholtz rollers (Goldstein \& Leib 1988, Goldstein \& Hultgren 1988).

The aim of the present paper is to propose a mathematical model which would describe nonlinear dynamics of CS, in particular the roll-up process. Prompted by the striking similarity between CS and instability waves in laminar shear layers (Ho \& Huerre 1990), the nonlinear critical-layer theory for the latter will be adapted to CS in turbulent flows. In doing so, two physical factors will be considered. The first is the enhanced non-parallelism associated with thicker turbulent shear flows. We shall choose the spreading rate, formally by taking a distinguished size of the mean turbulent Reynolds number, such that nonparallelism appears at leading order in the critical layer. This leads to, inter alia, a translating critical-layer effect. The second factor is the impact of small-scale fluctuations on CS through the phase-averaged Reynolds stresses. This is taken into account by using an eddy-viscosity type of closure model, one which allows for a phase lag, or relaxation time, between the Reynolds stresses and the strain rate of CS (Wu \& Zhou 1988).

The rest of the paper is organized as follows. Based on the triple decomposition of the instantaneous field, a general formulation is proposed in $\S 2$ for nonlinear dynamics of CS. Simple closure models are introduced for the time and phase-averaged Reynolds stresses of fine-scale turbulence, which influence the mean flow and CS respectively, respectively. $\S 3$ is devoted to developing an asymptotic nonlinear theory for CS, which is represented by a two-dimensional wavetrain of instability mode. The formulation starts by specifying in $\S 3.1$ the distinguished scalings that lead to a non-equilibrium and strongly nonlinear critical layer, where non-parallel-flow effects appear also at leading order. The disturbance in the main part of the shear layer is considered in $§ 3.2$; the analysis determines at leading order the eigen mode, and at the second order leads to a solvability condition consisting of an unknown jump across the critical layer. In $\S 3.3$, we analyse the disturbance in the critical layer to obtain the vorticity equation of the disturbance and the jump. Combined these with the solvability condition, we arrive at the key result of the present paper, namely, a system consisting of two coupled equations governing the nonlinear development of the 
amplitude and the vorticity of CS in the critical layer. In order to solve these equations, the appropriate 'initial' (upstream) and boundary conditions, consistent with the governing equations, are specified in $\S 3.4$ and $\S 3.5$ respectively. In $\S 4$, we analyse the Reynolds stress and the mean-flow distortion generated by nonlinear interactions of CS. The evolution system is solved numerically in $\S 5$, and numerical solutions are presented and compared with experiments. A summary and further discussions are given in $\S 6$.

\section{Formulation}

We consider a spatially developing incompressible turbulent free shear layer. The flow is to be described by Cartesian coordinates $(x, y, z)$, where $x, y$ and $z$ are in the streamwise, transverse and spanwise directions respectively. They are non-dimensionalized by $\delta_{0}^{*}$, the thickness of the shear layer at a typical location, which is to be specified later. The time $t$, the velocity $(u, v, w)$ and the pressure $p$ are normalized by $\delta_{0}^{*} / U_{0}^{*}, U_{0}^{*}$ and $\rho U_{0}^{* 2}$ respectively, where $U_{0}^{*}$ is a reference velocity and $\rho$ is the density of the fluid. The Reynolds number

$$
R=U_{0}^{*} \delta_{0}^{*} / \nu
$$

where $\nu$ is the kinematic molecular viscosity.

We begin by giving a general mathematical formulation for the nonlinear evolution of CS in a shear flow. For brevity, the Cartesian coordinates $(x, y, z)$ and the velocities $(u, v, w)$ will be denoted as $\mathbf{x}=\left(x_{1}, x_{2}, x_{3}\right)$ and $\mathbf{u}=\left(u_{1}, u_{2}, u_{3}\right)$, respectively.

\subsection{Flow decomposition and governing equations}

The instantaneous field $(\mathbf{u}, p)$ is composed of a mean flow $(\overline{\mathbf{U}}, \bar{P})$, quasi-periodic coherent motion $(\tilde{\mathbf{u}}, \tilde{p})$ representing CS and small-scale turbulence $\left(\mathbf{u}^{\prime}, p^{\prime}\right)$, and thus has the triple decomposition (Hussain \& Reynolds 1972)

$$
(\mathbf{u}, p)=(\overline{\mathbf{U}}, \bar{P})+(\tilde{\mathbf{u}}, \tilde{p})+\left(\mathbf{u}^{\prime}, p^{\prime}\right)
$$

where a quality with an overbar denotes the time average. CS can be extracted by the phase average, which may, for an instantaneous flow quantity $f(\mathbf{x}, t)$, be defined as

$$
<f>(\mathbf{x}, t) \equiv \lim _{N \rightarrow \infty} \frac{1}{N} \sum_{i}^{N} f\left(\mathbf{x}, t+t_{i}\right)=\lim _{N \rightarrow \infty} \frac{1}{N} \sum_{i}^{N} f(\mathbf{x}, t+i T),
$$

where $t_{i}$ denotes the instants at which the signal, or the structure, is deemed to have the same phase with respect to a reference oscillation (Hussain 1983); the second relation holds only when structures appear at regular interval $T$ (Hussain \& Reynolds 1970), which is the case where CS are introduced by controlled periodic excitation. The signature of CS, $\tilde{f}$, is then obtained as $\tilde{f} \equiv<f>-\bar{f}$.

The total time-averaged mean flow is driven by the Reynolds stresses contributed by both CS and turbulence. Unlike the conventional treatment, here we take $(\overline{\mathbf{U}}, \bar{P})$ to be the 'partial mean flow' driven only by the Reynolds stresses of the latter, that is, they satisfy the Reynolds-averaged equations

$$
\frac{\partial \bar{U}_{j}}{\partial x_{j}}=0, \quad \bar{U}_{j} \frac{\partial \bar{U}_{i}}{\partial x_{j}}=-\frac{\partial \bar{P}}{\partial x_{i}}+\frac{1}{R} \frac{\partial^{2} \bar{U}_{i}}{\partial x_{j} \partial x_{j}}-\frac{\partial}{\partial x_{j}} \overline{u_{i}^{\prime} u_{j}^{\prime}}
$$

This implies that the mean-flow distortion caused by nonlinear interactions of CS is to appear as part of CS. As we will explain later, this treatment is necessary. 
Substituting (2.2) into the Navier-Stokes equations and performing phase averaging, we obtain, after use is made of (2.4), the equations governing CS,

$$
\begin{gathered}
\frac{\partial \tilde{u}_{j}}{\partial x_{j}}=0 \\
\frac{\partial \tilde{u}_{i}}{\partial t}+\bar{U}_{j} \frac{\partial \tilde{u}_{i}}{\partial x_{j}}+\tilde{u}_{j} \frac{\partial \bar{U}_{i}}{\partial x_{j}}=-\frac{\partial \tilde{p}}{\partial x_{i}}+\frac{1}{R} \frac{\partial^{2} \tilde{u}_{i}}{\partial x_{j} \partial x_{j}}-\frac{\partial}{\partial x_{j}}\left(\tilde{u}_{i} \tilde{u}_{j}\right)+\frac{\partial \tilde{\tau}_{i j}}{\partial x_{j}}
\end{gathered}
$$

where

$$
\tilde{\tau}_{i j}=-\left[<u_{i}^{\prime} u_{j}^{\prime}>-\overline{u_{i}^{\prime} u_{j}^{\prime}}\right]
$$

are the phase-averaged Reynolds stresses of fine-scale turbulence; the latter affects CS through $\tilde{\tau}_{i j}$. The system is to be closed by introducing suitable models for $\tilde{\tau}_{i j}$ and $\bar{\tau}_{i j} \equiv$ $-\overline{u_{i}^{\prime} u_{j}^{\prime}}$.

The above formulation presents no difficulty if a reliable model for $\overline{u_{i}^{\prime} u_{j}^{\prime}}$ allows the 'partial mean flow' to be computed numerically. A problem does however arise if a mean field needs to be specified before hand. The measured velocity profile may not be appropriate because it is the total mean flow. A simple resolution is to resort to experiments where no artificial excitation is introduced and CS are relatively weak so that the corresponding mean flow may be regarded as approximating the partial mean flow. The measured mean flow may be used in the above formulation to investigate the evolution of CS artificially excited subsequently. In this case, the viewpoint of 'instability of a turbulent flow' may be taken, leading to an alternative formulation as follows. Suppose that an unforced turbulent flow is $(\overline{\mathbf{U}}, \bar{P})+\left(\mathbf{u}^{\prime}, p^{\prime}\right)$. To this 'turbulent base flow', coherent perturbations are then introduced in a controlled manner. The resulting perturbed turbulent flow has a triple decomposition

$$
(\overline{\mathbf{U}}, \bar{P})+(\tilde{\mathbf{u}}, \tilde{p})+\left(\mathbf{u}^{\prime \prime}, p^{\prime \prime}\right) .
$$

Note that introduction of CS affects also small-scale turbulence, which is now denoted by $\left(\mathbf{u}^{\prime \prime}, p^{\prime \prime}\right)$. Following the same steps, we can derive the equations

$$
\frac{\partial \tilde{u}_{i}}{\partial t}+\bar{U}_{j} \frac{\partial \tilde{u}_{i}}{\partial x_{j}}+\tilde{u}_{j} \frac{\partial \bar{U}_{i}}{\partial x_{j}}=-\frac{\partial \tilde{p}}{\partial x_{i}}+\frac{1}{R} \frac{\partial^{2} \tilde{u}_{i}}{\partial x_{j} \partial x_{j}}-\frac{\partial}{\partial x_{j}}\left(\tilde{u}_{i} \tilde{u}_{j}\right)+\frac{\partial \tilde{\tau}_{i j}^{\dagger}}{\partial x_{j}}
$$

where

$$
\tilde{\tau}_{i j}^{\dagger}=-\left[<u_{i}^{\prime \prime} u_{j}^{\prime \prime}>-\overline{u_{i}^{\prime} u_{j}^{\prime}}\right] .
$$

When CS are weak or have moderate amplitude, introduction of them may not significantly influence small-scale fluctuations, that is, $\mathbf{u}^{\prime \prime}$ remains close to $\mathbf{u}^{\prime}$ (whose magnitude is of $\mathrm{O}(1)$ ), or in a statistical sense $\overline{u_{i}^{\prime \prime} u_{j}^{\prime \prime}} \approx \overline{u_{i}^{\prime} u_{j}^{\prime}}$, and the two viewpoints, embodied in $(2.6)$ and (2.8), are then equivalent.

Note that in the formulation of Hussain \& Reynolds (1972), ( $\overline{\mathbf{U}}, \bar{P})$ represents the total mean flow. As a result, $\overline{\tilde{u}_{i} \tilde{u}_{j}}$ is added to $\overline{u_{i}^{\prime} u_{j}^{\prime}}$ in (2.4), and correspondingly $\tilde{u}_{i} \tilde{u}_{j}$ in (2.6) and (2.8) is replaced by $\left(\tilde{u}_{i} \tilde{u}_{j}-\overline{\tilde{u}_{i} \tilde{u}_{j}}\right)$, namely,

$$
\frac{\partial \tilde{u}_{i}}{\partial t}+\bar{U}_{j} \frac{\partial \tilde{u}_{i}}{\partial x_{j}}+\tilde{u}_{j} \frac{\partial \bar{U}_{i}}{\partial x_{j}}=-\frac{\partial \tilde{p}}{\partial x_{i}}+\frac{1}{R} \frac{\partial^{2} \tilde{u}_{i}}{\partial x_{j} \partial x_{j}}-\frac{\partial}{\partial x_{j}}\left(\tilde{u}_{i} \tilde{u}_{j}-\overline{\tilde{u}}_{i} \tilde{u}_{j}\right)+\frac{\partial \tilde{\tau}_{i j}}{\partial x_{j}} .
$$

The three formulations, (2.6), (2.8) and (2.9), are equally valid if suitable models can be provided for both the time- and phase-averaged Reynolds stresses, $-\overline{u_{i}^{\prime} u_{j}^{\prime}}$ and $\tilde{\tau}_{i j}$ (or $\tilde{\tau}_{i j}^{\dagger}$ ) of fine-grained turbulence. From the modelling perspective, the first and third seem 
advantageous since $\tilde{\tau}_{i j}$ should be less problematic to model than $\tilde{\tau}_{i j}^{\dagger}$, which involve smallscale turbulence of both perturbed and unperturbed states. In practice, further progress could be made by using an assumed mean flow, and it is better to predict (a) the meanflow correction by coherent fluctuations as part of CS, and (b) its impact on the evolution of CS rather than presuming that (a) and (b) are taken into account implicitly by the time-averaged mean flow. We shall therefore proceed with the first viewpoint, i.e. with the system (2.6)-(2.7).

\subsection{Closure models for the Reynolds stresses of small-scale turbulence}

Small-scale fluctuations contribute to time- and phase-averaged Reynolds stresses. No reliable closure model is available for either of them. Given the local nature of fine-grained turbulence, gradient type of models will be employed. For the time-averaged Reynolds stresses, the model is, in the non-dimensional form, written as

$$
\bar{\tau}_{i j}=-\overline{u_{i}^{\prime} u_{j}^{\prime}}=-\frac{2}{3} k \delta_{i j}+\frac{\nu_{t}}{R_{T}}\left(\partial \bar{U}_{i} / \partial x_{j}+\partial \bar{U}_{j} / \partial x_{i}\right),
$$

where $k=\frac{1}{2} \overline{\mathbf{u}^{\prime} \cdot \mathbf{u}^{\prime}}$ is the kinetic energy with $\delta_{i j}$ being the Kronecker delta, $\nu_{t}$ is the mean eddy viscosity normalized by a reference value $\nu_{t}^{*}$, and the mean turbulent Reynolds number $R_{T}$ is defined as

$$
R_{T}=U_{0}^{*} \delta_{0}^{*} / \nu_{t}^{*}
$$

The phase-averaged Reynolds stresses are time dependent, and they are related to the time-dependent strain rate of CS by a gradient type of model that includes the effect of time relaxation, namely,

$$
\tilde{\tau}_{i j}=-\left[<u_{i}^{\prime} u_{j}^{\prime}>-\overline{u_{i}^{\prime} u_{j}^{\prime}}\right]=\frac{\tilde{\nu}_{t}}{\tilde{R}_{T}}\left(\partial \tilde{u}_{i} / \partial x_{j}+\partial \tilde{u}_{j} / \partial x_{i}\right)(\mathbf{x}, t-\hat{\tau}),
$$

where $\tilde{\nu}_{t}$ is an eddy viscosity accounting for the impact of small-scale turbulence on CS, it is normalized by a reference value $\tilde{\nu}_{t}^{*}$ and the associated Reynolds number

$$
\tilde{R}_{T}=U_{0}^{*} \delta_{0}^{*} / \tilde{\nu}_{t}^{*}
$$

The key difference of (2.10) from an usual eddy viscosity model (cf. Hussain \& Reynolds 1972 ) is that it allows for a possible time delay $\hat{\tau}$ between the phase-averaged Reynolds stresses and the strain rate. Let $\widehat{\tau}_{i j}$ and $\widehat{u}$ denote the Fourier transforms of the $\tilde{\tau}_{i j}$ and $\tilde{\mathbf{u}}$ with respect to time $t$ respectively. Then the model (2.10) in spectral space can be expressed as

$$
\widehat{\tau}_{i j}(\mathbf{x}, \omega)=-\frac{\tilde{\nu}_{t}}{\tilde{R}_{T}} \mathrm{e}^{-\mathrm{i} \omega \hat{\tau}}\left(\partial \widehat{u}_{i} / \partial x_{j}+\partial \widehat{u}_{j} / \partial x_{i}\right)(\mathbf{x}, \omega),
$$

which consists of a complex eddy viscosity $\tilde{\nu}_{t} \mathrm{e}^{-\mathrm{i} \omega \hat{\tau}}$. This generalized eddy viscosity was first introduced by Wu \& Zhou (1989) in their study of CS in turbulent boundary layers. In general, both $\nu_{t}$ and $\tilde{\nu}_{t}$ may depend on space variables and/or the mean-flow (Reynolds \& Hussain 1972, Kitsios et al. 2010). In what follows, for simplicity they are assumed to be constants, in which case we may set $\nu_{t}=\tilde{\nu}_{t}=1$.

Use of (2.10) in (2.4) and (2.6) leads to

$$
\begin{gathered}
\frac{\partial \bar{U}_{j}}{\partial x_{j}}=0, \quad \bar{U}_{j} \frac{\partial \bar{U}_{i}}{\partial x_{j}}=-\frac{\partial}{\partial x_{i}}\left(\bar{P}+\frac{2}{3} k\right)+\left(\frac{1}{R}+\frac{1}{R_{T}}\right) \frac{\partial^{2} \bar{U}_{i}}{\partial x_{j} \partial x_{j}} \\
\frac{\partial \tilde{u}_{i}}{\partial t}+\bar{U}_{j} \frac{\partial \tilde{u}_{i}}{\partial x_{j}}+\tilde{u}_{j} \frac{\partial \bar{U}_{i}}{\partial x_{j}}=-\frac{\partial \tilde{p}}{\partial x_{i}}+\frac{1}{R} \frac{\partial^{2} \tilde{u}_{i}}{\partial x_{j} \partial x_{j}}+\frac{1}{\tilde{R}_{T}} \frac{\partial^{2} \tilde{u}_{i}(\mathbf{x}, t-\hat{\tau})}{\partial x_{j} \partial x_{j}}-\frac{\partial}{\partial x_{j}} \tilde{u}_{i} \tilde{u}_{j} .
\end{gathered}
$$


The above closure model accounts for the influence of small-scale fluctuations on CS (and the mean flow), but the fine-grained turbulence itself remains dynamically passive. As a result, the evolution of CS becomes, in a nutshell, analogous to that of waves propagating through a random medium.

\section{Asymptotic theory for nonlinear evolution of coherent structures}

The model proposed in the previous section is now applied to free shear layers. In order to present a self-consistent asymptotic description of the nonlinear development of the disturbance, we assume that $R \gg 1$ and $R_{T} \gg 1$ but $R_{T} \ll R$, i.e. the mean eddy viscosity is much greater than the molecular viscosity. The mean flow is two-dimensional and may be written as

$$
(\overline{\mathbf{U}}, \bar{P})=\left(\bar{U}_{B}(y, \tilde{x}), R_{T}^{-1} \bar{V}_{B}(y, \tilde{x}), P_{B}\right),
$$

where the slow streamwise variable

$$
\tilde{x}=x / R_{T} .
$$

Since $R_{T} \ll R$, the variation of the mean flow in the streamwise direction is therefore much faster than in the laminar case, implying enhanced non-parallelism. Substituting into (2.11) and making the usual boundary-layer approximation, we obtain the equations for $\bar{U}_{B}$ and $\bar{V}_{B}$,

$$
\frac{\partial \bar{U}_{B}}{\partial \tilde{x}}+\frac{\partial \bar{V}_{B}}{\partial y}=0, \quad \bar{U}_{B} \frac{\partial \bar{U}_{B}}{\partial \tilde{x}}+\bar{V}_{B} \frac{\partial \bar{U}_{B}}{\partial y}=\frac{\partial^{2} \bar{U}_{B}}{\partial y^{2}} .
$$

The focus of the present study will be on CS in the form of spanwise vortices (i.e. Kelvin-Helmholtz rollers), which are predominantly two-dimensional (Brown \& Roshko 1974, Wygnanski et al. 1979). It is then possible and convenient to introduce a streamfunction $\tilde{\psi}$. Elimination of the pressure term in (2.12) yields the equation

$$
\left(\frac{\partial}{\partial t}+\bar{U}_{B} \frac{\partial}{\partial x}+\frac{\bar{V}_{B}}{R_{T}} \frac{\partial}{\partial y}\right) \nabla^{2} \tilde{\psi}-\bar{U}_{B}^{\prime \prime} \frac{\partial \tilde{\psi}}{\partial x}-\frac{1}{R} \nabla^{4} \tilde{\psi}(\mathbf{x}, t)-\frac{1}{\tilde{R}_{T}} \nabla^{4} \tilde{\psi}(\mathbf{x}, t-\hat{\tau})=J\left(\tilde{\psi}, \nabla^{2} \tilde{\psi}\right),
$$

where $J(\psi, \varphi)$ is the Jacobian operator. Note that among high-order terms we have only retained the $O\left(R_{T}^{-1}\right)$ term of third-order derivative, which becomes important in the critical layer, whereas terms of the same order but of lower-order derivative are omitted since they remain negligible in the entire flow.

\subsection{Asymptotic scalings}

Prompted by the overwhelming experimental evidence, we subscribe to the view that CS in free shear layers behave as instability modes that develop from some small-amplitude disturbances upstream. We consider a two-dimensional wavetrain whose carrier wave has a frequency $\omega_{n}$ say. The carrier wave is locally neutral at a streamwise location $\tilde{x}=x_{n}$. The reference length $\delta_{0}^{*}$ is taken to be the shear-layer thickness at this location, which is chosen to be the origin of the coordinate for convenience. The precise meaning of $\delta_{0}^{*}$ will transpire in $\S 5.2$, where $\delta_{0}^{*}$ is related to the well-defined and experimentally measurable momentum thickness. The wavetrain becomes, following the initial exponential growth, neutrally stable in the vicinity of $x_{n}$ and enters a nonlinear stage due to enhanced nonlinearity associated with emergence of a critical layer as in the laminar flows (Goldstein 1995, 
Cowley \& Wu 1994). Since the critical level is at the inflection point and the perturbation is planar, the critical layer is strongly nonlinear and non-equilibrium, and has a thickness of $O\left(\epsilon^{1 / 2}\right)$, where $\epsilon \ll 1$ represents the amplitude.

The formulation will be presented for a plane mixing layer. However, the nonlinear critical-layer dynamics to be described is generic, independent of the detailed profile because the critical layer is thin. The analysis and resulting theory can easily be adjusted to other free shear layers such as wake and (planar and circular) jets. Indeed, the fact that the same nonlinear critical-layer dynamics operates explains why CS in these flows exhibit similar behaviours.

In the main part of the shear layer, the disturbance can, to leading order, be written as

$$
\epsilon A^{\dagger}(\bar{x}, \tau) \phi_{1}(y) \mathrm{e}^{\mathrm{i} \alpha \zeta}, \quad \zeta=x-c t,
$$

where $\alpha, c$ and $\phi_{1}$ are the wavenumber, phase speed and eigenfunction of the locally neutral mode respectively, and $A^{\dagger}$ is the amplitude function of the slow spatial and time variables, $\bar{x}$ and $\tau$, defined respectively as

$$
\bar{x}=\epsilon^{\frac{1}{2}} c^{-1} x, \quad \tau=\epsilon^{\frac{1}{2}} t,
$$

to describe the spatial and temporal modulation of the disturbance (Wu \& Tian 2012). For $\bar{x}=O(1)$, the CS corresponds to a nearly neutral instability mode (or more generally a wavetrain) residing on the turbulent mean flow. The effects of (molecular and eddy) viscosities and nonlinearity are negligible in the main part of the shear layer, but they all come into play in the critical layer if we choose the distinguished scaling, $R=O\left(\tilde{R}_{T}\right)=$ $O\left(\epsilon^{-3 / 2}\right)$. These may be expressed more precisely by writing

$$
R^{-1}=\bar{\lambda} \epsilon^{3 / 2}, \quad \tilde{R}_{T}^{-1}=\tilde{\lambda} \epsilon^{3 / 2},
$$

where $\bar{\lambda}=O(1)$ and $\tilde{\lambda}=O(1)$ are parameters measuring the importance of molecular and eddy viscosities relative to nonlinearity respectively (cf. Benney \& Bergeron 1969, Haberman 1972). Crucially, we take $R_{T}=O\left(R^{2 / 3}\right)$, that is,

$$
\sigma R_{T}=R^{2 / 3}
$$

with $\sigma$ being $O(1)$, so that the non-parallel-flow effect appears as well at leading order in the critical layer.

It follows from (3.2), (3.5) and (3.7) that the variables $\bar{x}$ and $\tilde{x}$ are related to each other via

$$
\tilde{x}=\left(\epsilon^{1 / 2} R_{T}\right)^{-1} c \bar{x}=\epsilon^{1 / 2}\left(\sigma \bar{\lambda}^{2 / 3} c\right) \bar{x} .
$$

In the vicinity of $x_{n}=0$, the mean-flow profile, $\bar{U}_{B}$ and $\bar{V}_{B}$ in (3.1), is expanded as

$$
\left(\bar{U}_{B}(y, \tilde{x}), \bar{V}_{B}(y, \tilde{x})\right)=(\bar{U}(y), \bar{V}(y))+\epsilon^{1 / 2}\left(\sigma \bar{\lambda}^{2 / 3} c\right) \bar{x}\left(\bar{U}_{1}(y), \bar{V}_{1}(y)\right)+\ldots,
$$

where $\left(\bar{U}_{1}, \bar{V}_{1}\right) \equiv \partial\left(\bar{U}_{B}, \bar{V}_{B}\right) / \partial \tilde{x}$ evaluated at $\tilde{x}=x_{n}=0$.

The derivation of the evolution system involves using simultaneously the multi-scale method and matched-asymptotic-expansion technique to analyse the disturbance outside and within the critical layer. The procedure is similar to those in Goldstein \& Hultgren (1988) and Wu \& Tian (2012), but the main difference is that non-parallelism now appears at leading order in the critical layer. The reader who is interested primarily in physical aspects of the problem may skip the detail, and go directly to the final outcome of the analysis, which is the nonlinear evolution system consisting of the amplitude equation (3.24) for $A^{\dagger}$ coupled with a nonlinear equation (3.23) for the critical-layer vorticity. The reader is also reminded that the scalings and critical-layer dynamics are different for threedimensional disturbances such as a single (Churilov \& Shukhman 1994), or a pair of oblique modes (see e.g. Goldstein \& Choi 1989, Wu, Cowley \& Lee 1993). 


\subsection{Outer expansion}

In the main part of the shear layer, the stream function $\tilde{\psi}$ expands

$$
\tilde{\psi}=\epsilon A^{\dagger}(\bar{x}, \tau) \phi_{1}(y) \mathrm{e}^{\mathrm{i} \alpha \zeta}+\epsilon^{\frac{3}{2}} \sum_{m=1}^{\infty} \phi_{2}^{(m)}(y ; \bar{x}, \tau) \mathrm{e}^{\mathrm{i} m \alpha \zeta}+\epsilon^{2} \sum_{m=0}^{\infty} \phi_{3}^{(m)}(y ; \bar{x}, \tau) \mathrm{e}^{\mathrm{i} m \alpha \zeta}+\text { c.c. }+\ldots
$$

The eigenfunction $\phi_{1}$ is determined by the eigenvalue problem,

$$
\mathcal{L}(\alpha) \phi_{1}=0, \quad \phi_{1} \rightarrow 0 \text { as } y \rightarrow \pm \infty
$$

where the Rayleigh operator

$$
\mathcal{L}(\alpha)=\left(\frac{\partial^{2}}{\partial y^{2}}-\alpha^{2}\right)-\frac{\bar{U}^{\prime \prime}}{\bar{U}-c}
$$

Let $y_{c}$ denote the critical level, i.e. $\bar{U}\left(y_{c}\right)=c$. As $\hat{\eta} \equiv y-y_{c} \rightarrow 0, \phi_{1}$ has the asymptote,

$$
\phi_{1} \sim 1+\frac{1}{2}\left(\alpha^{2}+\frac{\bar{U}_{c}^{\prime \prime \prime}}{\bar{U}_{c}^{\prime}}\right) \hat{\eta}^{2}+a_{1} \hat{\eta}+\ldots,
$$

where $a_{1}$ is a constant. The function $\phi_{2}^{(1)}$ is governed by the system consisting of inhomogeneous Rayleigh equation and the boundary condition,

$$
\left.\begin{array}{c}
\mathcal{L}(\alpha) \phi_{2}^{(1)}=-2 \mathrm{i} \alpha c^{-1} \frac{\partial A^{\dagger}}{\partial \bar{x}} \phi_{1}-(\mathrm{i} \alpha)^{-1} \mathcal{D}_{0} A^{\dagger} \frac{\bar{U}^{\prime \prime}}{(\bar{U}-c)^{2}} \phi_{1} \\
-\left(\sigma \bar{\lambda}^{2 / 3} c\right) \bar{x} A^{\dagger} \frac{\bar{U}^{\prime \prime} \bar{U}_{1}-(\bar{U}-c) \bar{U}_{1}^{\prime \prime}}{(\bar{U}-c)^{2}} \phi_{1}, \\
\phi_{2}^{(1)} \rightarrow 0 \quad \text { as } \quad y \rightarrow \pm \infty
\end{array}\right\}
$$

where we have introduced the differential operator

$$
\mathcal{D}_{0}=\frac{\partial}{\partial \tau}+\frac{\partial}{\partial \bar{x}}
$$

As $\hat{\eta} \rightarrow 0$, the solution is, by examining the asymptotic properties of both sides of (3.12), found to behave as

$$
\phi_{2}^{(1)} \sim b_{2}^{(1)}+a_{2}^{(1) \pm} \hat{\eta}+\left(\mathrm{i} \alpha \bar{U}_{c}^{\prime}\right)^{-1} \frac{\bar{U}_{c}^{\prime \prime \prime}}{\bar{U}_{c}^{\prime}}\left\{-\mathcal{D}_{0} A^{\dagger}+\mathrm{i} \chi_{2} \bar{x} A^{\dagger}\right\} \hat{\eta} \log |\hat{\eta}|+O\left(\hat{\eta}^{2}\right)
$$

where $b_{2}^{(1)}$ and $a_{2}^{(1) \pm}$ are as yet unknown functions of $\bar{x}$, and

$$
\chi_{2}=\alpha\left(\sigma \bar{\lambda}^{2 / 3} c\right)\left(\bar{U}_{c}^{\prime} \bar{U}_{1, c}^{\prime \prime}-\bar{U}_{c}^{\prime \prime \prime} \bar{U}_{1, c}\right) / \bar{U}_{c}^{\prime \prime \prime}
$$

The jump $\left(a_{2}^{(1)+}-a_{2}^{(1)-}\right)$ is to be determined by analysing the critical-layer dynamics. For the boundary-value problem (3.12) to have a solution, a (modified) solvability condition

$$
a_{2}^{(1)+}-a_{2}^{(1)-}=-\mathrm{i} \alpha^{-1} J_{2} \frac{\partial A^{\dagger}}{\partial \tau}+\mathrm{i}\left(2 \alpha c^{-1} J_{1}-\alpha^{-1} J_{2}\right) \frac{\partial A^{\dagger}}{\partial \bar{x}}+\left(\sigma \bar{\lambda}^{2 / 3} c\right) I_{1} \bar{x} A^{\dagger}
$$

must be satisfied, which is derived via a routine procedure: multiply $\phi_{1}$ to both sides of the inhomogeneous Rayleigh equation in (3.12), perform integration by parts on the 
left-hand side but take into account of the jump across the critical layer by using (3.14) (cf. Redekopp 1977); in (3.16),

$$
\begin{gathered}
J_{1}=\int_{-\infty}^{\infty} \phi_{1}^{2} \mathrm{~d} y, \quad J_{2}=f_{-\infty}^{\infty} \frac{\bar{U}^{\prime \prime}}{(\bar{U}-c)^{2}} \phi_{1}^{2} \mathrm{~d} y, \\
I_{1}=f_{-\infty}^{\infty} \frac{\bar{U}^{\prime \prime} \bar{U}_{1}-(\bar{U}-c) \bar{U}_{1}^{\prime \prime}}{(\bar{U}-c)^{2}} \phi_{1}^{2} \mathrm{~d} y,
\end{gathered}
$$

with $J_{2}$ and $I_{1}$ both being Cauchy's principal values.

The solution for $\phi_{2}^{(1)}$ could of course only be determined up to an arbitrary multiple of the eigenfunction $\phi_{1}$. The multiplier of $\phi_{1}$ is a function of $\bar{x}$, and can be fixed by imposing the condition that $\phi_{2}^{(1)}$ be orthogonal to $\phi_{1}$. This is however unnecessary in the present study as the indeterminacy does not affect the dynamics of the CS, or the Reynolds stresses and the mean-flow distortion to be considered in $\S 4$.

The harmonic components $\phi_{2}^{(m)}$ satisfy homogeneous Rayleigh equations

$$
\mathcal{L}(m \alpha) \phi_{2}^{(m)}=0 \quad(m=2,3, \ldots),
$$

and as $\hat{\eta} \rightarrow 0$,

$$
\phi_{2}^{(m)} \sim b_{2}^{(m)}+a_{2}^{(m) \pm} \hat{\eta}+O\left(\hat{\eta}^{2}\right) .
$$

It should be reminded that $\phi_{2}^{(m)}(m \neq 1)$ are not eigenfunctions because there is a forcing from the interaction within the critical layer through the jumps $\left(a_{2}^{(m)+}-a_{2}^{(m)-}\right)$, which renders the boundary-value problem inhomogeneous. An important fact of a strong nonlinear critical layer is that all harmonics are generated at the same order, which causes vortex to roll up.

\subsection{Inner expansion: critical layer analysis}

The dynamics of CS within the critical layer can be analysed by introducing the local transverse variable

$$
Y=\hat{\eta} / \epsilon^{\frac{1}{2}}=\left(y-y_{c}\right) / \epsilon^{\frac{1}{2}} .
$$

The mean-flow quantities are expanded as Taylor series of $Y$, namely,

$$
\left.\begin{array}{rl}
\bar{U}(y, 0) & =c+\bar{U}_{c}^{\prime} \epsilon^{1 / 2} Y+\frac{1}{6} \bar{U}_{c}^{\prime \prime \prime} \epsilon^{3 / 2} Y^{3}+\ldots, \\
\bar{U}_{1}(y, 0) & =\bar{U}_{1, c}+\bar{U}_{1, c}^{\prime} \epsilon^{1 / 2} Y+\frac{1}{2} \bar{U}_{1, c}^{\prime \prime} \epsilon Y^{2}+\ldots, \\
\bar{V}(y, 0) & =\bar{V}_{c}+\bar{V}_{c}^{\prime} \epsilon^{1 / 2} Y+\ldots
\end{array}\right\}
$$

The stream function of the perturbation, $\tilde{\psi}$, expands as

$$
\tilde{\psi}=\epsilon \Psi_{0}+\epsilon^{\frac{3}{2}} \Psi_{1}+\epsilon^{2} \ln \epsilon^{\frac{1}{2}} \Psi_{2 L}+\epsilon^{2} \Psi_{2}+\epsilon^{5 / 2} \Psi_{3}+\ldots
$$

The first three terms are just the straightforward continuation of the outer solution, namely,

$$
\left.\begin{array}{rl}
\Psi_{0} & =A^{\dagger} \mathrm{e}^{\mathrm{i} \alpha \zeta}+c . c ., \\
\Psi_{1} & =\sum b_{2}^{(m)} \mathrm{e}^{\mathrm{i} m \alpha \zeta}+a_{1} Y A^{\dagger} \mathrm{e}^{\mathrm{i} \alpha \zeta}+c . c ., \\
\Psi_{2 L} & =\frac{\mathrm{i} \bar{U}_{c}^{\prime \prime \prime}}{\alpha \bar{U}_{c}^{\prime 2}}\left[\mathcal{D}_{0} A^{\dagger}-\mathrm{i} \chi_{2} \bar{x} A^{\dagger}\right] Y \mathrm{e}^{\mathrm{i} \alpha \zeta}+c . c .,
\end{array}\right\}
$$


and it can easily be verified that they all satisfy the required equations.

Substituting expansions (3.20) and (3.21) with (3.22) into (3.4), we obtain the equation for $\Psi_{2}$. That equation can, by introducing

$$
\Omega^{\dagger}=\Psi_{2, Y Y}-\left(\alpha^{2}+\frac{\bar{U}_{c}^{\prime \prime \prime}}{\bar{U}_{c}^{\prime}}\right)\left(A^{\dagger} \mathrm{e}^{\mathrm{i} \alpha \zeta}+\text { c.c. }\right)
$$

be simplified to

$$
\mathcal{L}_{N} \Omega^{\dagger}-\bar{\lambda} \frac{\partial^{2}}{\partial Y^{2}} \Omega^{\dagger}-\tilde{\lambda} \frac{\partial^{2}}{\partial Y^{2}} \Omega^{\dagger}(t-\hat{\tau})=\frac{\bar{U}_{c}^{\prime \prime \prime}}{\bar{U}_{c}^{\prime}}\left\{-\mathcal{D}_{0}+\mathrm{i} \chi_{2} \bar{x}\right\}\left(A^{\dagger} \mathrm{e}^{\mathrm{i} \alpha \zeta}+c . c .\right),
$$

where the nonlinear operator

$$
\mathcal{L}_{N}=\mathcal{D}_{0}+\left[\bar{U}_{c}^{\prime} Y+\left(\sigma \bar{\lambda}^{2 / 3} c \bar{U}_{1, c}\right) \bar{x}\right] \frac{\partial}{\partial \zeta}-\left(\mathrm{i} \alpha A^{\dagger} \mathrm{e}^{\mathrm{i} \alpha \zeta}+\text { c.c. }-\sigma \bar{\lambda}^{\frac{2}{3}} \bar{V}_{c}\right) \frac{\partial}{\partial Y} .
$$

Matching with the outer solution for the fundamental component determines the jump $\left(a_{2}^{(1)+}-a_{2}^{(1)-}\right)$, which is inserted into (3.16) to give the evolution equation for the amplitude $A^{\dagger}$,

$\frac{\alpha}{2 \pi} \int_{-\infty}^{\infty} \int_{0}^{2 \pi / \alpha} \Omega^{\dagger} \mathrm{e}^{-\mathrm{i} \alpha \zeta} \mathrm{d} \zeta \mathrm{d} Y=-\mathrm{i} \alpha^{-1} J_{2} \frac{\partial A^{\dagger}}{\partial \tau}+\mathrm{i}\left(2 \alpha c^{-1} J_{1}-\alpha^{-1} J_{2}\right) \frac{\partial A^{\dagger}}{\partial \bar{x}}+\left(\sigma \bar{\lambda}^{2 / 3} c\right) I_{1} \bar{x} A^{\dagger}$.

Equations (3.23) and (3.24) form the system that governs the nonlinear evolution of the disturbance.

Matching the harmonic components determines the jump

$$
a_{2}^{(m)+}-a_{2}^{(m)-}=\frac{\alpha}{2 \pi} \int_{-\infty}^{\infty} \int_{0}^{2 \pi / \alpha} \Omega^{\dagger} \mathrm{e}^{-\mathrm{i} m \alpha \zeta} \mathrm{d} \zeta \mathrm{d} Y .
$$

Once $\Omega^{\dagger}$ is known, the harmonics in the main part of the shear layer can be obtained by solving (3.18) subject to (3.19) and (3.25).

It is convenient to introduce the normalised dependent and independent variables

$$
\bar{A}=\alpha^{2} \bar{U}_{c}^{\prime} A^{\dagger}, \quad \Omega=\Omega^{\dagger}\left(\alpha \bar{U}_{c}^{\prime}\right)^{2} / \bar{U}_{c}^{\prime \prime \prime}, \quad \bar{\zeta}=\alpha \zeta,
$$

as well as the (normalized) local transverse coordinate centred at the moving critical level,

$$
\bar{\eta}=\alpha \bar{U}_{c}^{\prime}\left(Y+\left(\sigma \bar{\lambda}^{2 / 3} c \bar{U}_{1, c} / \bar{U}_{c}^{\prime}\right) \bar{x}\right) .
$$

The system (3.23)-(3.24) then becomes

$$
\left.\begin{array}{c}
{\left[\frac{\partial}{\partial \tau}+\frac{\partial}{\partial \bar{x}}+\bar{\eta} \frac{\partial}{\partial \bar{\zeta}}-\left(\mathrm{i} \bar{A} \mathrm{e}^{\mathrm{i} \bar{\zeta}}+c . c .-\chi\right) \frac{\partial}{\partial \bar{\eta}}-\bar{\lambda}_{1} \frac{\partial^{2}}{\partial \bar{\eta}^{2}}\right] \Omega-\bar{\lambda}_{2} \frac{\partial^{2}}{\partial \bar{\eta}^{2}} \Omega(t-\hat{\tau})} \\
=\left[-\left(\frac{\partial}{\partial \tau}+\frac{\partial}{\partial \bar{x}}\right)+\mathrm{i} \chi_{2} \bar{x}\right]\left(\bar{A} \mathrm{e}^{\mathrm{i} \bar{\zeta}}+\text { c.c. }\right), \\
\frac{1}{2 \pi} \int_{-\infty}^{\infty} \int_{0}^{2 \pi} \Omega \mathrm{e}^{-\mathrm{i} \bar{\zeta}} \mathrm{d} \bar{\zeta} \mathrm{d} \bar{\eta}=\Lambda_{1} \frac{\partial \bar{A}}{\partial \tau}+\Lambda_{2} \frac{\partial \bar{A}}{\partial \bar{x}}+\Lambda_{0} \bar{x} \bar{A},
\end{array}\right\}
$$

where we have put

$$
\chi=\alpha \sigma \bar{\lambda}^{2 / 3}\left(c \bar{U}_{1, c}+\bar{U}_{c}^{\prime} \bar{V}_{c}\right)
$$




$$
\begin{gathered}
\bar{\lambda}_{1}=\left(\alpha \bar{U}_{c}^{\prime}\right)^{2} \bar{\lambda}, \quad \bar{\lambda}_{2}=\left(\alpha \bar{U}_{c}^{\prime}\right)^{2} \tilde{\lambda} \\
\Lambda_{1}=-\mathrm{i} J_{2} \frac{\bar{U}_{c}^{\prime 2}}{\bar{U}_{c}^{\prime \prime \prime}}, \quad \Lambda_{2}=\mathrm{i}\left(2 \alpha^{2} c^{-1} J_{1}-J_{2}\right) \frac{\bar{U}_{c}^{\prime 2}}{\bar{U}_{c}^{\prime \prime \prime}}, \quad \Lambda_{0}=\alpha\left(\sigma \bar{\lambda}^{\frac{2}{3}} c\right) \frac{\bar{U}_{c}^{\prime 2}}{\bar{U}_{c}^{\prime \prime \prime}} I_{1} .
\end{gathered}
$$

As the unknown amplitude function $\bar{A}$ appears in the coefficient of the partial differential equation governing the vorticity, the critical-layer dynamics is 'strongly nonlinear'.

The parameter $\chi$ characterises the effect of a translating critical layer, which was first identified by Cowley (1985) and Haynes \& Cowley (1986) for certain time-dependent flows. For the present problem, this effect arises because non-parallelism causes the critical level to drift with respect to the streamwise variable $\bar{x}$ at the same rate as that at which the amplitude evolves. Non-parallelism is associated with the streamwise variation of the streamwise velocity and with the transverse velocity itself, of the mean flow. The former alters directly the growth rate through the terms proportional to $\bar{x}$ in (3.28), while both contribute to the translating critical-layer effect as (3.29) indicates.

\subsection{Upstream condition}

Let us now consider what condition should be imposed upstream as an 'initial condition'. In the upstream limit $\bar{x} \rightarrow-\infty$, the disturbance is small so that the nonlinear term in the first equation of (3.28) can be neglected, and the resulting linear equation admits the solution

$$
\Omega \sim\left\{-\int_{0}^{\infty}\left[\mathcal{D}_{0}-\mathrm{i} \chi_{2}(\bar{x}-\xi)\right] \bar{A}(\bar{x}-\xi, \tau-\xi) \mathrm{e}^{-\frac{1}{3} \lambda_{1} \xi^{3}+\frac{1}{2} \mathrm{i} \chi \xi^{2}-\mathrm{i} \bar{\eta} \xi} \mathrm{d} \xi\right\} \mathrm{e}^{\mathrm{i} \bar{\zeta}}+\text { c.c. },
$$

where

$$
\lambda_{1}=\bar{\lambda}_{1}+\bar{\lambda}_{2} \mathrm{e}^{\mathrm{i} \alpha c \hat{\tau}}
$$

It follows that

$$
\int_{-\infty}^{\infty} \Omega \mathrm{d} \bar{\eta}=-\pi\left(\mathcal{D}_{0}-\mathrm{i} \chi_{2} \bar{x}\right) \bar{A} \mathrm{e}^{\mathrm{i} \bar{\zeta}}
$$

This result is inserted to the second equation of (3.28) to give

$$
\frac{\partial \bar{A}}{\partial \bar{x}}+c_{g}^{-1} \frac{\partial \bar{A}}{\partial \tau}=\sigma_{s} \bar{x} \bar{A}
$$

where the group velocity

$$
c_{g}=\left(\Lambda_{2}+\pi\right) /\left(\Lambda_{1}+\pi\right)=1-2 \alpha^{2} c^{-1} J_{1} /\left\{\frac{\mathrm{i} \pi \bar{U}_{c}^{\prime \prime \prime}}{\bar{U}_{c}^{\prime}\left|\bar{U}_{c}^{\prime}\right|}+J_{2}\right\},
$$

and the coefficient

$$
\sigma_{s}=\frac{\mathrm{i} \pi \chi_{2} \bar{U}_{c}^{\prime}-\Lambda_{0}\left|\bar{U}_{c}^{\prime}\right|}{\pi \bar{U}_{c}^{\prime}+\Lambda_{2}\left|\bar{U}_{c}^{\prime}\right|}
$$

It can be deduced from (3.33) that

$$
\bar{A} \rightarrow \mathrm{e}^{\sigma_{s} \bar{x}^{2} / 2+\kappa \bar{x}-\mathrm{i} S_{0} \tau} \quad \text { as } \quad \bar{x} \rightarrow-\infty,
$$

where $\kappa=\mathrm{i} S_{0} / c_{g}$; here $S_{0}$ measures the deviation of the disturbance frequency $(\alpha c+$ $\epsilon^{1 / 2} S_{0}$ ) from that of the neutral mode (and is equivalent to $S_{1}$ in Goldstein \& Leib (1988)). Note that since $c_{g}$ is a complex number (which is expected since the mode considered is neutral rather than being locally most unstable), it is impossible to introduce the coordinate moving with the group velocity. As such the modulation equation is of first-order rather than second-order as would be the case if the group velocity is real. Note that the linear growth rate is unaffected by the effect of a translating critical layer; the latter remains dormant in the linear regime and only comes into play in the nonlinear stage. 


\subsection{Fourier decomposition and boundary conditions}

The solution for $\bar{A}$ and $\Omega$ can be written as a Fourier series (cf. Goldstein \& Leib 1988)

$$
\bar{A}=A \mathrm{e}^{-\mathrm{i} S_{0} \tau}, \quad \Omega=\sum_{n=-\infty}^{\infty} Q_{n}(\tau, \bar{x}, \eta) \mathrm{e}^{\mathrm{i} n\left(\bar{\zeta}-S_{0} \tau\right)} \quad\left(Q_{-n}=Q_{n}^{*}\right)
$$

where $\eta=\bar{\eta}-S_{0}$. Then $Q_{n}(n=0,1,2, \ldots)$, among which $Q_{0}$ represents the mean-flow distortion generated by the nonlinear interaction of CS, satisfy the system of coupled equations,

$$
\begin{gathered}
\left(\mathcal{D}_{0}+\mathrm{i} n \eta+\chi \frac{\partial}{\partial \eta}-\lambda_{n} \frac{\partial^{2}}{\partial \eta^{2}}\right) Q_{n}+\mathrm{i} \frac{\partial}{\partial \eta}\left(A^{*} Q_{n+1}-A Q_{n-1}\right)=-\delta_{n 1}\left(\mathcal{D}_{0}-\mathrm{i} S_{0}-\mathrm{i} \chi_{2} \bar{x}\right) A \\
\int_{-\infty}^{\infty} Q_{1} \mathrm{~d} \eta=-\mathrm{i} S_{0} \Lambda_{1} A+\Lambda_{1} \frac{\partial A}{\partial \tau}+\Lambda_{2} \frac{\partial A}{\partial \bar{x}}+\Lambda_{0} \bar{x} A
\end{gathered}
$$

where $\delta_{n 1}$ denotes the Kronecker delta, and $\lambda_{n}$ is a complex quantity

$$
\lambda_{n}=\bar{\lambda}_{1}+\bar{\lambda}_{2} \mathrm{e}^{\mathrm{i} n \omega \hat{\tau}} .
$$

It follows from (3.38) that as $\eta \rightarrow \pm \infty$,

$$
\begin{gathered}
Q_{1} \rightarrow\left\{\frac{\mathrm{i}}{\eta}-\frac{\mathcal{D}_{0}}{\eta^{2}}-\frac{\mathrm{i} \mathcal{D}_{0}^{2}}{\eta^{3}}+\frac{\chi}{\eta^{3}}\right\}\left(\mathcal{D}_{0}-\mathrm{i} S_{0}-\mathrm{i} \chi_{2} \bar{x}\right) A+O\left(\eta^{-4}\right) \\
Q_{0} \rightarrow-\frac{|A|^{2}}{\eta^{2}}+\frac{2 \mathrm{i}}{\eta^{3}}\left\{A \mathcal{D}_{0} A^{*}-A^{*} \mathcal{D}_{0} A+\mathrm{i} S_{0}|A|^{2}+\mathrm{i} \chi_{2} \bar{x}|A|^{2}+\mathrm{i}\left(\chi_{2}+\chi\right) B_{1}\right\}+O\left(\eta^{-4}\right) \\
Q_{2} \rightarrow-\frac{\mathrm{i}}{2 \eta^{3}} A\left(\mathcal{D}_{0}-\mathrm{i} S_{0}-\mathrm{i} \chi_{2} \bar{x}\right) A+O\left(\eta^{-4}\right)
\end{gathered}
$$

where we have put

$$
B_{1}(\bar{x}, \tau)=\int_{0}^{\infty}|A|^{2}(\bar{x}-\xi, \tau-\xi) \mathrm{d} \xi .
$$

Due to the slow decay of $Q_{1}$, the integral in (3.39) must be interpreted as a Cauchy principal value. The infinite domain in the $\eta$-direction is truncated to a large but finite interval $-M \leq \eta \leq M$. Then (3.39) can be written as

$$
\left(\Lambda_{0} \bar{x}-\mathrm{i} S_{0} \Lambda_{1}\right) A+\Lambda_{1} \frac{\partial A}{\partial \tau}+\Lambda_{2} \frac{\partial A}{\partial \bar{x}}=I_{10}-\frac{2}{M} \mathcal{D}_{0}\left(\mathcal{D}_{0}-\mathrm{i} S_{0}-\mathrm{i} \chi_{2} \bar{x}\right) A+O\left(M^{-3}\right),
$$

where we have defined

$$
I_{n k}=\int_{-M}^{M} \eta^{k} Q_{n} \mathrm{~d} \eta
$$

The second-order derivative with respect to $\bar{x}$ in the above equation is inconvenient for numerical integration. Following a similar procedure to that in Wu \& Tian (2012), we obtain a first-order system with respect to $\bar{x}$,

$$
\begin{aligned}
& \tilde{\Lambda}_{1} \frac{\partial A}{\partial \tau}+\tilde{\Lambda}_{2} \frac{\partial A}{\partial \bar{x}}-\tilde{\Lambda}_{d, 1} \frac{\partial^{2} A}{\partial \tau^{2}}-\tilde{\Lambda}_{d, 2} \frac{\partial^{2} A}{\partial \tau \partial \bar{x}}+\tilde{\Lambda}_{0} A \\
& \quad=\left(q_{1}-q_{2} \bar{x}-\frac{2 \Lambda_{d}}{M \Lambda_{2}} \frac{\partial}{\partial \tau}\right) I_{10}+\frac{2 \mathrm{i}}{M} I_{11}-\frac{4}{M^{2} \Lambda_{2}}\left(I_{12}+\mathrm{i} \chi I_{10}-A^{*} I_{20}\right)
\end{aligned}
$$

the derivation and the expressions for the coefficients are given in the appendix A. The equation is subject to the initial condition

$$
A \rightarrow \mathrm{e}^{\sigma_{s} \bar{x}^{2} / 2+\kappa \bar{x}} \quad \text { as } \bar{x} \rightarrow-\infty
$$




\section{Reynolds stress and the mean-flow distortion induced by CS}

The self-interaction of the CS produces Reynolds stresses, through which a mean-flow distortion is generated. Two reasons may be given for retaining this distortion as part of the coherent motion. First, the distortion evolves over the same streamwise length scale as the amplitude of the CS does, and this scale is much shorter than that of the background mean flow developing under the action of molecular diffusion and small-scale turbulence. Second, when the CS is a temporally modulated packet, the induced distortion is time-dependent, and hence should not be treated as part of a mean field. However, as far as its impact on the nonlinear development of the CS is concerned, the modulated distortion plays the same role as the steady distortion in the special case of a singlefrequency disturbance, which means that the distortion, modulated or not, should be treated on the same footing, namely as part of the CS. We note that this viewpoint was taken in the theory of Cohen et al. (1994).

The mean-flow distortion induced by an instability mode in a laminar mixing layer with a tanh profile was analysed by Goldstein \& Leib (1988). The present analysis is for an arbitrary profile, and will lead to a more general and complete result.

\subsection{The Reynolds stresses of CS}

The characteristics of Reynolds stresses is of interest in its own right and is now considered. In order to calculate them, the streamwise and transverse velocities of the CS are obtained, to $O\left(\epsilon^{3 / 2}\right)$ accuracy, in terms of the stream function:

$$
\left.\begin{array}{l}
\tilde{u}=\epsilon A^{\dagger} \phi_{1}^{\prime} \mathrm{e}^{\mathrm{i} \alpha \zeta}+\epsilon^{3 / 2}\left[\phi_{2, y}^{(1)} \mathrm{e}^{\mathrm{i} \alpha \zeta}+\ldots\right], \\
\tilde{v}=-\epsilon(\mathrm{i} \alpha) A^{\dagger} \phi_{1} \mathrm{e}^{\mathrm{i} \alpha \zeta}-\epsilon^{3 / 2}\left[\left(\mathrm{i} \alpha \phi_{2}^{(1)}+c^{-1} A_{\bar{x}}^{\dagger} \phi_{1}\right) \mathrm{e}^{\mathrm{i} \alpha \zeta}+\ldots\right],
\end{array}\right\}
$$

where only the fundamental component is needed and written out. The general solution for $\phi_{2}^{(1)}$, which is governed by (3.12), can be written as (Wu \& Tian 2012)

$$
\phi_{2}^{(1)}=B^{\dagger} \phi_{1}+C^{ \pm} \mathcal{F}_{0}-2 \mathrm{i} \alpha c^{-1} A_{\bar{x}}^{\dagger} \mathcal{F}_{1}-(\mathrm{i} \alpha)^{-1} \mathcal{D}_{0} A^{\dagger} \mathcal{F}_{2}-\left(\sigma \lambda^{2 / 3} c\right) \bar{x} A^{\dagger} \mathcal{F}_{3},
$$

where $B^{\dagger}(\bar{x}, \tau)$ is a function of $\bar{x}$ and $\tau$, and

$$
\begin{gathered}
\mathcal{F}_{0}=\phi_{1} \int_{y_{c}}^{y} \frac{1}{\phi_{1}^{2}} \mathrm{~d} y \\
\mathcal{F}_{1}=\phi_{1} \int_{y_{c}}^{y} \frac{1}{\phi_{1}^{2}} \mathrm{~d} \tilde{y} \int_{\infty}^{\tilde{y}} \phi_{1}^{2}(\xi) \mathrm{d} \xi, \quad \mathcal{F}_{2}=\phi_{1} \int_{y_{c}}^{y} \frac{1}{\phi_{1}^{2}} \mathrm{~d} \tilde{y} \int_{\infty}^{\tilde{y}} \frac{\bar{U}^{\prime \prime} \phi_{1}^{2}}{(\bar{U}-c)^{2}} \mathrm{~d} \xi, \\
\mathcal{F}_{3}=\phi_{1} \int_{y_{c}}^{y} \frac{1}{\phi_{1}^{2}} \mathrm{~d} \tilde{y} \int_{\infty}^{\tilde{y}} \frac{\left[\bar{U}^{\prime \prime} \bar{U}_{1}-(\bar{U}-c) \bar{U}_{1}^{\prime \prime}\right] \phi_{1}^{2}}{(\bar{U}-c)^{2}} \mathrm{~d} \xi .
\end{gathered}
$$

In order for $\phi_{2}^{(1)}$ to satisfy the boundary conditions that $\phi_{2}^{(1)} \rightarrow 0$ as $y \rightarrow \pm \infty$, we have to set

$$
C^{+}=0, \quad C^{-}=-2 \mathrm{i} \alpha c^{-1} J_{1} A_{\bar{x}}^{\dagger}-(\mathrm{i} \alpha)^{-1} J_{2} \mathcal{D}_{0} A^{\dagger}-\left(\sigma \lambda^{2 / 3} c\right) \bar{x} A^{\dagger} I_{1} .
$$

Taking the limit $y \rightarrow y_{c}$, the solution (4.2) assumes the asymptote (3.14) with

$$
b_{2}^{(1)}=B^{\dagger},
$$




$$
a_{2}^{(1) \pm}=C^{ \pm}+a_{1} B^{\dagger}-2 \mathrm{i} \alpha c^{-1} A_{\bar{x}}^{\dagger} \int_{\infty}^{y_{c}} \phi_{1}^{2} \mathrm{~d} y-(\mathrm{i} \alpha)^{-1} \mathcal{D}_{0} A^{\dagger} J_{0}-\left(\sigma \lambda^{2 / 3} c\right) \bar{x} A^{\dagger} I_{0},
$$

where

$$
\begin{aligned}
& J_{0}= \int_{\infty}^{\hat{a}} \frac{\bar{U}^{\prime \prime} \phi_{1}^{2}}{(\bar{U}-c)^{2}} \mathrm{~d} y+\int_{\hat{a}}^{y_{c}}\left[\frac{\bar{U}^{\prime \prime} \phi_{1}^{2}}{(\bar{U}-c)^{2}}-\frac{\bar{U}_{c}^{\prime \prime \prime}}{\bar{U}_{c}^{\prime 2}\left(y-y_{c}\right)}\right] \mathrm{d} y-\frac{\bar{U}_{c}^{\prime \prime \prime}}{\bar{U}_{c}^{\prime 2}} \ln \left|\hat{a}-y_{c}\right|, \\
& I_{0}=\int_{\infty}^{\hat{a}} \frac{\left[\bar{U}^{\prime \prime} \bar{U}_{1}-(\bar{U}-c) \bar{U}_{1}^{\prime \prime}\right] \phi_{1}^{2}}{(\bar{U}-c)^{2}} \mathrm{~d} y \\
& \quad+\int_{\hat{a}}^{y_{c}}\left[\frac{\left[\bar{U}^{\prime \prime} \bar{U}_{1}-(\bar{U}-c) \bar{U}_{1}^{\prime \prime}\right] \phi_{1}^{2}}{(\bar{U}-c)^{2}}-\frac{\chi_{1}}{y-y_{c}}\right] \mathrm{d} y-\chi_{1} \ln \left|\hat{a}-y_{c}\right|,
\end{aligned}
$$

with $\hat{a} \neq y_{c}$ being an arbitrary constant and $\chi_{1}=\left(\bar{U}_{c}^{\prime \prime \prime} \bar{U}_{1, c}-\bar{U}_{c}^{\prime} \bar{U}_{1, c}^{\prime \prime}\right) / \bar{U}_{c}^{\prime 2}$.

Of interest is the shear stress component $\tau_{12} \equiv-\left(\tilde{u} \tilde{v}^{*}+\right.$ c.c. $)$. For a nearly neutral mode, the leading-order term vanishes, leaving us with

$$
\tau_{12}=-\epsilon^{5 / 2}\left[A^{\dagger}\left(\mathrm{i} \alpha \phi_{2}^{(1) *}-c^{-1} A_{\bar{x}}^{\dagger *} \phi_{1}\right) \phi_{1}^{\prime}+\mathrm{i} \alpha A^{\dagger *} \phi_{1} \phi_{2, y}^{(1)}+\text { c.c. }\right] .
$$

The first two terms in (4.4) are regular, while the third term, or rather $\phi_{2, y}^{(1)}$ in it, exhibits a logarithmic singularity at $y_{c}$. In order to find an expression for the Reynolds stress valid in the entire shear layer, $\phi_{2, y}^{(1)}$ has to be replaced by a composite solution, $\left(\phi_{2, y}^{(1)}\right)_{c}$, which is constructed as follows. First, the inner solution for the $O\left(\epsilon^{3 / 2}\right)$ velocity is

$$
U_{3 / 2} \equiv a_{2}^{(1)-}+\bar{U}_{c}^{\prime \prime \prime} /\left(\alpha \bar{U}_{c}^{\prime}\right)^{3}\left\{\lim _{M \rightarrow \infty}\left[\mathrm{i}\left(\mathcal{D}_{0}-\mathrm{i} \chi_{2} \bar{x}\right) A \ln |M|+\int_{-M}^{\eta} Q_{1} \mathrm{~d} \eta\right]\right\} .
$$

By using the additive rule, the composite solution is constructed as

$$
\left(\phi_{2, y}^{(1)}\right)_{c}=\phi_{2, y}^{(1)}+\left(U_{3 / 2}-a_{2}^{(1) \pm}\right)-\frac{\mathrm{i} \bar{U}_{c}^{\prime \prime \prime}}{\alpha \bar{U}_{c}^{\prime 2}}\left(\mathcal{D}_{0}-\mathrm{i} \chi_{2} \bar{x}\right) A^{\dagger} \ln |\eta|
$$

where the last term is the common part of $\phi_{2, y}^{(1)}$ and $U_{3 / 2}$ in the limits of $y \rightarrow y_{c}$ and $\eta \rightarrow \infty$ respectively. It is important to note that the undetermined complementary solution $B^{\dagger} \phi_{1}$ in (4.2) does not contribute to the Reynolds stress, neither does the last term. Taking this into account and inserting (4.2) and (4.5) into (4.4), we can, without the need for the complete solution for $\phi_{2}^{(1)}$, determine fully the Reynolds stress,

$$
\begin{array}{r}
\tau_{12}=c^{-1} \frac{\partial\left|A^{\dagger}\right|^{2}}{\partial \bar{x}}\left\{\phi_{1} \phi_{1}^{\prime}+2 \alpha^{2} \phi_{1}^{\prime}\left(\mathcal{F}_{1}+\hat{C}^{ \pm} \mathcal{F}_{0}\right)-c \phi_{1}^{\prime}\left(\mathcal{F}_{2}+\hat{C}^{ \pm} \mathcal{F}_{0}\right)-2 \alpha^{2} \phi_{1}\left(\mathcal{F}_{1}^{\prime}+\hat{C}^{ \pm} \mathcal{F}_{0}^{\prime}\right)\right. \\
\left.+c \phi_{1}\left[\mathcal{F}_{2}^{\prime}+\hat{C}^{ \pm} \mathcal{F}_{0}^{\prime}\right]\right\}-\left[\mathrm{i} \alpha A^{\dagger *} \phi_{1}\left(U_{3 / 2}-a_{2}^{(1) \pm}-\frac{\mathrm{i} \bar{U}_{c}^{\prime \prime \prime}}{\alpha \bar{U}_{c}^{\prime 2}} A_{\bar{x}}^{\dagger} \ln |\eta|\right)+\text { c.c. }\right],
\end{array}
$$

where we have put $\hat{C}^{+}=0$ and $\hat{C}^{-}=1$. Since the last term in (4.6) is confined within the critical layer, in the majority of the shear layer the Reynolds stress $\tau_{12}$ is proportional to $\partial\left|A^{\dagger}\right|^{2} / \partial \bar{x}$ and would therefore change its sign when the CS evolves through its peak amplitude as was observed in experiments (WW, Marasli et al. 1992). 


\subsection{The mean-flow distortion}

The mean-flow distortion in the main part of the shear layer has a $O\left(\epsilon^{2}\right)$ streamwise velocity, whereas that in the critical layer has a larger, $O\left(\epsilon^{3 / 2}\right)$, streamwise velocity due to the larger gradient of the Reynolds stress there. Despite being weaker, the mean-flow distortion in the main part makes the dominant contribution to the change in the shearlayer thickness. The solution takes the form $\epsilon^{2}\left(u_{M}, \epsilon^{1 / 2} v_{M}, p_{M}\right)$ with $u_{M}$ and $v_{M}$ being related to $\phi_{3}^{(0)}$ in $(3.8)$

$$
u_{M}=\phi_{3, y}^{(0)}, \quad v_{M}=-c^{-1} \phi_{3, \bar{x}}^{(0)} .
$$

The governing equations are found as

$$
\left.\begin{array}{c}
c^{-1} \frac{\partial u_{M}}{\partial \bar{x}}+\frac{\partial v_{M}}{\partial y}=0, \quad \frac{\partial p_{M}}{\partial y}=-\frac{\partial}{\partial y}\left(2 \alpha^{2}\left|A^{\dagger}\right|^{2} \phi_{1}^{2}\right), \\
{\left[\frac{\partial}{\partial \tau}+(\bar{U} / c) \frac{\partial}{\partial \bar{x}}\right] u_{M}+v_{M} \bar{U}^{\prime}=-c^{-1} \frac{\partial p_{M}}{\partial \bar{x}}-\left[2 c^{-1} \frac{\partial\left|A^{\dagger}\right|^{2}}{\partial \bar{x}} \phi_{1}^{\prime 2}-\frac{\partial \tau_{12}}{\partial y}\right] .}
\end{array}\right\}
$$

From the transverse momentum equation we find that

$$
p_{M}=-2 \alpha^{2}\left|A^{\dagger}\right|^{2} \phi_{1}^{2} .
$$

In terms of the stream function $\phi_{3}^{(0)}$, the streamwise momentum equation in (4.7) can be written as

$$
\left[\frac{\partial}{\partial \tau}+(\bar{U} / c) \frac{\partial}{\partial \bar{x}}\right] \frac{\partial \phi_{3}^{(0)}}{\partial y}-\left(\bar{U}^{\prime} / c\right) \frac{\partial \phi_{3}^{(0)}}{\partial \bar{x}}=-S
$$

where

$$
S=c^{-1} \frac{\partial\left|A^{\dagger}\right|^{2}}{\partial \bar{x}}\left(\phi_{1}^{\prime 2}-\phi_{1} \phi_{1}^{\prime \prime}-2 \alpha^{2} \phi_{1}^{2}\right)+\mathrm{i} \alpha A^{\dagger}\left(\phi_{1}^{\prime \prime} \phi_{2}^{(1) *}-\phi_{1} \phi_{2, y y}^{(1) *}\right)+c . c .
$$

A numerical approach is needed when the disturbance is a modulated wave packet. For a simple wave, it turns out that $S$ can be written as

$$
S=c^{-1} \frac{\partial\left|A^{\dagger}\right|^{2}}{\partial \bar{x}} \hat{S}(y)
$$

with the shape function

$$
\begin{gathered}
\hat{S}(y)=\left(\phi_{1}^{\prime 2}-\phi_{1} \phi_{1}^{\prime \prime}-2 \alpha^{2} \phi_{1}^{2}\right)-\phi_{1}^{\prime \prime}\left[2 \alpha^{2}\left(\mathcal{F}_{1}+\hat{C}^{ \pm} \mathcal{F}_{0}\right)-c\left(\mathcal{F}_{2}+\hat{C}^{ \pm} \mathcal{F}_{0}\right)\right] \\
+\phi_{1}\left[2 \alpha^{2}\left(\mathcal{F}_{1}^{\prime \prime}+\hat{C}^{ \pm} \mathcal{F}_{0}^{\prime \prime}\right)-c\left(\mathcal{F}_{2}^{\prime \prime}+\hat{C}^{ \pm} \mathcal{F}_{0}^{\prime \prime}\right)\right]
\end{gathered}
$$

Once again, the expression (4.10) explains the experimental observation that the Reynolds stress (in the main part of the shear flow) switches its sign at the location where the CS reaches its peak amplitude and starts to attenuate. Equation (4.9) reduces to

$$
\bar{U} \frac{\partial \phi_{3}^{(0)}}{\partial y}-\bar{U}^{\prime} \phi_{3}^{(0)}=-\hat{S}
$$

and the solution is found as

$$
\phi_{3}^{(0)}=\left|A^{\dagger}\right|^{2} \bar{U}\left[a_{3}^{(0) \pm}-\int_{ \pm \infty}^{y} \frac{\hat{S}}{\bar{U}^{2}} \mathrm{~d} y\right]
$$

where $a_{3}^{(0) \pm}$ are unknown constants. Let $\bar{U} \rightarrow \bar{U}_{ \pm}$as $y \rightarrow \pm \infty$, Then

$$
\phi_{3}^{(0)} \rightarrow a_{3}^{(0) \pm} \bar{U}_{ \pm}\left|A^{\dagger}\right|^{2} \text { as } y \rightarrow \pm \infty,
$$


which implies that the mean-flow distortion spreads to the outer invisicd layer, where the streamwise and transverse velocities both become $O\left(\epsilon^{5 / 2}\right)$, and a pressure of the same order of magnitude is induced. On introducing $\bar{y}=\epsilon^{1 / 2} y / c$, the perturbation may be written as $\epsilon^{5 / 2}\left(\hat{u}_{M}^{ \pm}, \hat{v}_{M}^{ \pm}, \hat{p}_{M}^{ \pm}\right)$, where the scaled velocity $\left(\hat{u}_{M}^{ \pm}, \hat{v}_{M}^{ \pm}\right)$and pressure $\hat{p}_{M}^{ \pm}$are functions of $\bar{x}$ and $\bar{y}$, and satisfy the equations

$$
\frac{\partial \hat{u}_{M}^{ \pm}}{\partial \bar{x}}+\frac{\partial \hat{v}_{M}^{ \pm}}{\partial \bar{y}}=0, \quad \bar{U}_{ \pm} \frac{\partial \hat{u}_{M}^{ \pm}}{\partial \bar{x}}=-\frac{\partial \hat{p}_{M}^{ \pm}}{\partial \bar{x}}, \quad \bar{U}_{ \pm} \frac{\partial \hat{v}_{M}^{ \pm}}{\partial \bar{x}}=-\frac{\partial \hat{p}_{M}^{ \pm}}{\partial \bar{y}} .
$$

It follows that

$$
\nabla^{2} \hat{p}_{M}^{ \pm}=0,\left.\quad \frac{\partial \hat{p}_{M}^{ \pm}}{\partial \bar{y}}\right|_{\bar{y}=0}=c^{-1} \bar{U}_{ \pm}^{2} a_{3}^{(0) \pm} \frac{\partial^{2}\left|A^{\dagger}\right|^{2}}{\partial \bar{x}^{2}}(-\infty<\bar{x}<\infty),
$$

where the boundary condition corresponds to the matching with the transverse velocity $v_{M}=-c^{-1} \phi_{3, \bar{x}}^{(0)}$ at the upper and lower outer edges of the shear layer. Clearly, in the lower stream, if $\bar{y}$ is changed to $-\bar{y}>0$, the Laplace equation for $\hat{p}_{M}^{-}$remains invariant, but the boundary condition changes its sign, that is,

$$
\nabla^{2} \hat{p}^{-}=0,\left.\quad \frac{\partial \hat{p}^{-}}{\partial \bar{y}}\right|_{\bar{y}=0}=-c^{-1} \bar{U}_{-}^{2} a_{3}^{(0)-} \frac{\partial^{2}\left|A^{\dagger}\right|^{2}}{\partial \bar{x}^{2}}(-\infty<\bar{x}<\infty) .
$$

In the main part of the shear layer, there exists an induced mean pressure, $\epsilon^{5 / 2} p_{M}^{(2)}$, with $p_{M}^{(2)}$ satisfying

$$
\frac{\partial p_{M}^{(2)}}{\partial y}=-\frac{\partial}{\partial y}\left(2 \mathrm{i} \alpha A^{\dagger *} \phi_{1} \phi_{2}^{(1)}+\text { c.c. }\right) .
$$

Integration across the shear layer gives

$$
p_{M}^{(2)}(\infty)-p_{M}^{(2)}(-\infty)=0 .
$$

It follows by matching that $\hat{p}_{M}^{+}=\hat{p}_{M}^{-}$at $\bar{y}=0$, and hence

$$
a_{3}^{(0)+} \bar{U}_{+}^{2}=-a_{3}^{(0)-} \bar{U}_{-}^{2} .
$$

On the other hand, as $y \rightarrow y_{c}^{ \pm}$it follows from (4.13) that

$$
\begin{gathered}
\phi_{3}^{(0)} \rightarrow \bar{U}_{c}\left[a_{3}^{(0) \pm}+\frac{\bar{U}_{c}^{\prime \prime \prime}}{\bar{U}_{c} \bar{U}_{c}^{\prime 2}} \ln \left|y-y_{c}\right|-I_{s}^{ \pm}\right]\left|A^{\dagger}\right|^{2} \\
\phi_{3, y}^{(0)} \rightarrow \bar{U}_{c}^{\prime}\left[a_{3}^{(0) \pm}-I_{s}^{ \pm}+\frac{\bar{U}_{c}^{\prime \prime \prime}}{\bar{U}_{c} \bar{U}_{c}^{\prime 2}} \ln \left|y-y_{c}\right|\right]\left|A^{\dagger}\right|^{2}+\frac{\bar{U}_{c}^{\prime \prime \prime}}{\bar{U}_{c}^{\prime 2}} \frac{\left|A^{\dagger}\right|^{2}}{y-y_{c}},
\end{gathered}
$$

where

$$
I_{s}^{ \pm}=\int_{ \pm \infty}^{\hat{a}^{ \pm}} \frac{\hat{S}}{\bar{U}^{2}} \mathrm{~d} y+\int_{\hat{a}^{ \pm}}^{y_{c}}\left[\frac{\hat{S}}{\bar{U}^{2}}+\frac{\bar{U}_{c}^{\prime \prime \prime} / \bar{U}_{c}^{\prime 2}}{\bar{U}_{c}\left(y-y_{c}\right)}\right] \mathrm{d} y+\frac{\bar{U}_{c}^{\prime \prime \prime}}{\bar{U}_{c} \bar{U}_{c}^{\prime 2}} \ln \left|y_{c}-\hat{a}^{ \pm}\right|
$$

with $\hat{a}^{+}>y_{c}$ and $\hat{a}^{-}<y_{c}$ being arbitrary constants.

The jump $\left(a_{3}^{(0)+}-a_{3}^{(0)-}\right)$ can be found by considering the mean-flow distortion $Q_{0}$ in the critical layer. Let $\Psi_{2}^{(0)}$ be the stream function related to $Q_{0}: \Psi_{2 Y Y}^{(0)}=\bar{U}_{c}^{\prime \prime \prime} /\left(\alpha \bar{U}_{c}^{\prime}\right)^{2} Q_{0}$. Using this in (3.38) for $n=0$ and integrating the resulting equation with respect to $\eta$, we obtain

$$
\left[\mathcal{D}_{0}+\chi \frac{\partial}{\partial \eta}-\lambda_{0} \frac{\partial^{2}}{\partial \eta^{2}}\right] \Psi_{2, \eta}^{(0)}=-\mathrm{i} \bar{U}_{c}^{\prime \prime \prime} /\left(\alpha \bar{U}_{c}^{\prime}\right)^{4}\left(A^{*} Q_{1}-A Q_{1}^{*}\right)
$$


Integrating once more and making use of (3.39), we find that

$$
\mathcal{D}_{0}\left[\Psi_{2}^{(0)}(\infty)-\Psi_{2}^{(0)}(-\infty)\right]=\left(\alpha^{2} \bar{U}_{c}^{\prime}\right)^{-2}\left[\left(2 \alpha^{2} c^{-1} J_{1}-J_{2}\right) \frac{\partial|A|^{2}}{\partial \bar{x}}-J_{2} \frac{\partial|A|^{2}}{\partial \tau}\right] .
$$

For a non-modulated disturbance, from the above relation the jump $\Psi_{2}^{(0)}(\infty)-\Psi_{2}^{(0)}(-\infty)$ is obtained, which must, by the matching principle, be equal to $\phi_{3}^{(0)}\left(y_{c}^{+}\right)-\phi_{3}^{(0)}\left(y_{c}^{-}\right)$, leading to the relation

$$
a_{3}^{(0)+}-a_{3}^{(0)-}-\left(I_{s}^{+}-I_{s}^{-}\right)=\left(2 \alpha^{2} c^{-1} J_{1}-J_{2}\right) / \bar{U}_{c} .
$$

It follows from (4.17) and (4.20) that

$$
a_{3}^{(0) \pm}= \pm \bar{U}_{\mp}^{2}\left[\left(I_{s}^{+}-I_{s}^{-}\right)+\left(2 \alpha^{2} c^{-1} J_{1}-J_{2}\right) / \bar{U}_{c}\right] /\left(\bar{U}_{+}^{2}+\bar{U}_{-}^{2}\right) .
$$

The mean-flow distortion in the critical layer is given by $\left(\epsilon^{3 / 2} U_{M}^{(1)}+\epsilon^{2} U_{M}^{(2)}\right)$ with

$$
U_{M}^{(1)}=\frac{\bar{U}_{c}^{\prime \prime \prime}}{\left(\alpha \bar{U}_{c}^{\prime}\right)^{3}} \int_{-\infty}^{\eta} Q_{0} \mathrm{~d} \eta
$$

and the $O\left(\epsilon^{2}\right)$ term $U_{M}^{(2)}$ is contained in $\Psi_{3}$ in (3.21), which consists, like $\Psi_{2}$, of all harmonics. Let the mean-flow component be denoted by $\Psi_{3}^{(0)}$. Then $U_{M}^{(2)}=\Psi_{3, Y}^{(0)}$. As in $\mathrm{Wu} \& \operatorname{Tian}(2012), \Psi_{3, Y Y}^{(0)} \sim O(1 / \eta)$ for $\eta \gg 1$, and so $U_{M}^{(2)} \rightarrow c_{M}|A|^{2} \ln |\eta|$ with $c_{M}=\bar{U}_{c}^{\prime \prime \prime} /\left(\alpha^{4} \bar{U}_{c} \bar{U}_{c}^{\prime 3}\right)$, matching to the corresponding logarithmic term in (4.19). The composite solution for the mean-flow distortion is then given by

$$
U_{M}=\epsilon^{3 / 2} U_{M}^{(1)}+\epsilon^{2}\left[\left(U_{M}^{(2)}-c_{M}|A|^{2} \ln |\eta|\right)+\epsilon^{2}\left[\phi_{3, y}^{(0)}-\frac{\bar{U}_{c}^{\prime \prime \prime} / \bar{U}_{c}^{\prime 2}}{\left(y-y_{c}\right)}\left|A^{\dagger}\right|^{2}\right] .\right.
$$

The width of the shear layer is usually measured by the momentum thickness, defined as

$$
\Theta=\frac{1}{4} \int_{-\infty}^{\infty}\left(U_{+}-\bar{U}^{\dagger}\right)\left(\bar{U}^{\dagger}-U_{-}\right) \mathrm{d} y
$$

where $\bar{U}^{\dagger}=\bar{U}+U_{M}$ is the perturbed mean velocity. At leading order, the definition gives the momentum thickness of the 'partial' (or unperturbed) mean flow,

$$
\theta=\frac{1}{4} \int_{-\infty}^{\infty}\left(U_{+}-\bar{U}\right)\left(\bar{U}-U_{-}\right) \mathrm{d} y
$$

The $O\left(\epsilon^{2}\right)$ change in the momentum thickness is then found as

$$
\Delta \Theta=-\frac{1}{2} \epsilon^{2} \int_{-\infty}^{\infty}(\bar{U}-c)\left\{\left[\phi_{3, y}^{(0)}-\frac{\bar{U}_{c}^{\prime \prime \prime} / \bar{U}_{c}^{\prime 2}}{y-y_{c}}\left|A^{\dagger}\right|^{2}\right]+\epsilon^{-1 / 2} U_{M}^{(1)}+\left(U_{M}^{(2)}-c_{M}|A|^{2} \ln |\eta|\right)\right\} \mathrm{d} y
$$

The contribution of the leading-order inner solution was ignored in Goldstein \& Leib (1988) on the ground that $U_{M}^{(1)}$ is trapped in the critical layer of $O\left(\epsilon^{1 / 2}\right)$ width, where the integrand, $\epsilon^{-1 / 2}(\bar{U}-c) U_{M}^{(1)}$, is of order one so that the contribution is of $O\left(\epsilon^{1 / 2}\right)$. Here it is retained in the spirit of composition approximation, but the even smaller term $U_{M}^{(2)}$ will be neglected. Using equation (4.12) and performing integration by parts, we find that

$$
\Delta \Theta=-\frac{1}{4} \epsilon^{2}\left\{\left[(1-c) \phi_{3}^{(0)}(\infty)+(1+c) \phi_{3}^{(0)}(-\infty)\right]+c\left[\phi_{3}^{(0)}\left(y_{c}^{+}\right)-\phi_{3}^{(0)}\left(y_{c}^{-}\right)\right]\right.
$$




$$
\begin{aligned}
& \left.-\int_{-\infty}^{\infty} S \mathrm{~d} y\right\}-\frac{1}{2} \epsilon^{2} \int_{-\infty}^{\infty}(\bar{U}-c)\left[-\frac{\bar{U}_{c}^{\prime \prime \prime} / \bar{U}_{c}^{\prime 2}}{y-y_{c}}\left|A^{\dagger}\right|^{2}+\epsilon^{-1 / 2} U_{M}^{(1)}\right] \mathrm{d} y \\
= & -\frac{1}{4} \epsilon^{2}\left|A^{\dagger}\right|^{2}\left[\left(a_{3}^{(0)+}-a_{3}^{(0)-}\right)-c^{2}\left(I_{s}^{+}-I_{s}^{-}\right)-\int_{-\infty}^{\infty} \hat{S} \mathrm{~d} y\right] \\
& -\frac{1}{2} \epsilon^{2} \int_{-\infty}^{\infty}\left[\bar{U}-\frac{1}{2}\left(\bar{U}_{+}+\bar{U}_{-}\right)\right]\left\{-\frac{\bar{U}_{c}^{\prime \prime \prime} / \bar{U}_{c}^{\prime 2}}{y-y_{c}}\left|A^{\dagger}\right|^{2}+\epsilon^{-1 / 2} U_{M}^{(1)}\right\} \mathrm{d} y .
\end{aligned}
$$

Substitution of (4.20) into the above gives final expression for $\Delta \Theta$,

$$
\begin{aligned}
\Delta \Theta= & -\frac{1}{4} \epsilon^{2}\left|A^{\dagger}\right|^{2}\left[\left(1-c^{2}\right)\left(I_{s}^{+}-I_{s}^{-}\right)+\left(2 \alpha^{2} c^{-1} J_{1}-J_{2}\right) / \bar{U}_{c}-\int_{-\infty}^{\infty} \hat{S} \mathrm{~d} y\right] \\
& -\frac{1}{2} \epsilon^{2} \int_{-\infty}^{\infty}\left[\bar{U}-\frac{1}{2}\left(\bar{U}_{+}+\bar{U}_{-}\right)\right]\left\{-\frac{\bar{U}_{c}^{\prime \prime \prime} / \bar{U}_{c}^{\prime 2}}{y-y_{c}}\left|A^{\dagger}\right|^{2}+\epsilon^{-1 / 2} U_{M}^{(1)}\right\} \mathrm{d} y .
\end{aligned}
$$

It is interesting that $\Delta \Theta$ can be determined without fixing the individual values of $a_{3}^{(0) \pm}$.

Equation (4.23) indicates that if the full profile is to be obtained accurate up to $O\left(\epsilon^{2}\right)$, the critical-layer equation at $O\left(\epsilon^{5 / 2}\right)$ has to be solved to calculate $\Psi_{3}$ and $\Psi_{3 Y}^{(0)}=U_{M}^{(2)}$. An $O\left(\epsilon^{2}\right)$ velocity jump across the critical layer is present in the outer solution $\phi_{3, y}^{(0)}$, and is smoothed out by the inner solution $U_{M}^{(2)}$. This can be shown by examining (but without solving) the equation for $\Psi_{3}$, which reads

$$
\begin{aligned}
\mathcal{L}_{N} \Psi_{3, Y Y}= & \mathcal{G}-c^{-1} \bar{U}_{1, c} \Psi_{2, Y Y \bar{x}}-\bar{U}_{1, c}^{\prime} Y \Psi_{2, Y Y \zeta}-\sigma \bar{\lambda}^{2 / 3}\left[\bar{V}_{c}^{\prime} Y+\left(\sigma \bar{\lambda}^{2 / 3} c\right) \bar{x} \bar{V}_{1, c}\right] \Psi_{2, Y Y Y} \\
& -\bar{U}_{1, c} \Psi_{1, \zeta \zeta}-\bar{U}_{1, c}^{\prime} Y \Psi_{0, \zeta \zeta}-2 c^{-1} \bar{U}_{1, c} \Psi_{0, \zeta \bar{x}}+\bar{U}_{1, c}^{\prime \prime}\left[\Psi_{1, \zeta}+c^{-1} \Psi_{0, \bar{x}}\right] \\
& +\bar{U}_{1, c}^{\prime \prime \prime} Y \Psi_{0, \zeta}+\frac{1}{2}\left(\sigma \bar{\lambda}^{2 / 3} c\right)^{2} \bar{x}^{2}\left[\bar{U}_{2}^{\prime \prime} \Psi_{0, \zeta}-\bar{U}_{2} \Psi_{0, \zeta \zeta}\right]
\end{aligned}
$$

where $\mathcal{G}$ stands for the right-hand side of (2.42) in Wu \& Tian (2012), and $\bar{U}_{2}=\partial^{2} \bar{U}_{B} / \partial \tilde{x}^{2}$ evaluated at $y_{c}$ and $x_{n}=0$. Integrating (4.27) with respect to $Y$ for the mean-flow component, and making use of the asymptotic behaviours of $\Psi_{2, Y}^{(0)}$ and $\Psi_{3, Y Y}^{(0)}$, we obtain

$$
\mathcal{D}_{0}\left[\Psi_{3, Y}^{(0)}(\infty)-\Psi_{3, Y}^{(0)}(-\infty)\right]=c^{-1} \bar{U}_{c}^{\prime} \frac{\partial}{\partial \bar{x}}\left[\Psi_{2}^{(0)}-Y \Psi_{2, Y}^{(0)}\right]_{-\infty}^{\infty}=\left.c^{-1} \bar{U}_{c}^{\prime} \frac{\partial \Psi_{2}^{(0)}}{\partial \bar{x}}\right|_{-\infty} ^{\infty} .
$$

For the non-modulated case, evaluation of the right-hand side by matching with (4.18) shows that

$$
U_{M}^{(2)}(\infty)-U_{M}^{(2)}(-\infty)=\bar{U}_{c}^{\prime}\left[a_{3}^{(0)+}-a_{3}^{(0)-}-\left(I_{s}^{+}-I_{s}^{-}\right)\right] .
$$

From this relation and (4.19) it follows that

$$
U_{M}^{(2)}(\infty)-U_{M}^{(2)}(-\infty)=\phi_{3, y}^{(0)}\left(y_{c}^{+}\right)-\phi_{3, y}^{(0)}\left(y_{c}^{-}\right)
$$

indicating that the jump in the streamwise velocity is smoothed out within the critical layer as expected.

The expressions for the Reynolds stress (4.6), the mean-flow distortion (4.23) and the change in the momentum thickness (4.26) are valid for a general profile $\bar{U}$. These quantities are of physical interest as they are relevant for understanding CS and/or their impact on the shear layer, and they will be calculated in $\S 5$ and presented after numerical solutions for the amplitude and vorticity of the CS are obtained. In performing the computation, use is to be made of the relation

$$
\eta=\alpha \bar{U}_{c}^{\prime}\left[\left(y-y_{c}\right) / \epsilon^{1 / 2}+\left(\sigma \lambda^{\overline{2} / 3} c \bar{U}_{1, c} / \bar{U}_{c}^{\prime}\right) \bar{x}\right]-S_{0} .
$$




\section{$5 \quad$ Numerical solutions for mixing layers}

\section{$5.1 \quad$ Numerical method}

The numerical work consists of solving the coupled evolution system (3.38) and (3.45) subject to the initial condition (3.46) and the boundary conditions (3.41)-(3.43). The system is truncated with $0 \leq n \leq N$ (where $n$ refers to the order of harmonics in (3.37)), and solved in a large but finite domain $-M \leq \eta \leq M$. A predictor-corrector method is employed to march downstream. The amplitude equation for $A$ is discretized using the fourth-order explicit (Adams-Bashforth) and implicit (Adams-Moulton) schemes to construct the predictor and corrector respectively. The integrals are evaluated using Simpson's rule. The vorticity equations for $Q_{n}$ are discretized by the Crank-Nicolson scheme.

\subsection{Mean velocity profile}

The theory is now applied to a mixing layer that forms between two uniform streams with velocities $U_{1}^{*}$ and $U_{2}^{*}<U_{1}^{*}$. The reference velocity $U_{0}^{*}=\frac{1}{2}\left(U_{1}^{*}-U_{2}^{*}\right)$. As in many previous studies, we will specify a mean-flow profile which is broadly in agreement with experiment data. The latter indicate that the mean-flow profile remains self-similar in the streamwise direction while its local thickness varies, and thus it takes the form

$$
\bar{U}_{B}=U_{c}+F\left(\eta^{\dagger}\right), \quad \eta^{\dagger}=\left[y-y_{0}(\tilde{x})\right] / \delta(\tilde{x}),
$$

where

$$
\bar{U}_{c}=\left(U_{1}^{*}+U_{2}^{*}\right) /\left(U_{1}^{*}-U_{2}^{*}\right)
$$

the function $F$ characterises the shape, while $y_{0}$ and $\delta$ represent the centre and thickness of the shear layer respectively. The dimensional momentum thickness $\theta^{*}$, which is often measured in experiments, is related to $\theta$ (as defined by (4.24)), $\delta$ and $\delta^{*}$ by

$$
\theta^{*}=\delta_{0}^{*} \theta=C_{1} \delta_{0}^{*} \delta=C_{1} \delta^{*}
$$

where for the similarity profile (5.1) the constant $C_{1}$ is found as

$$
C_{1}=\frac{1}{4} \int_{-\infty}^{\infty}\left(1-F^{2}\right) \mathrm{d} \eta^{\dagger}
$$

With a given $F$, the (rescaled) transverse velocity $V_{B}$, whose value at the critical level is needed in our theory, can be obtained from the continuity equation as will be shown below. Before prescribing the profile $F$, it is informative to discuss useful mean-flow properties which are independent of $F$. First, the streamwise variations of $y_{0}(\tilde{x})$ and $\delta(\tilde{x})$ are not independent but satisfy a constraint. We derive it by considering the momentum equation (3.3), which can be rewritten as

$$
\frac{\partial\left[\bar{U}_{B}\left(\bar{U}_{B}-\bar{U}_{ \pm}\right)\right]}{\partial \tilde{x}}+\frac{\partial\left[\bar{V}_{B}\left(\bar{U}_{B}-\bar{U}_{ \pm}\right)\right]}{\partial y}=\frac{\partial^{2} \bar{U}_{B}}{\partial y^{2}}
$$

where substraction of $\bar{U}_{ \pm}=\bar{U}_{c} \pm 1$ is for $y>0$ and $y<0$ respectively. Integration of the above equation with respect to $y$ gives

$$
\int_{-\infty}^{0} \bar{U}_{B}\left(\bar{U}_{B}-\bar{U}_{-}\right) d y+\int_{0}^{\infty} \bar{U}_{B}\left(\bar{U}_{B}-\bar{U}_{+}\right) d y=C_{0}
$$


where $C_{0}$ is a constant. The relation corresponds to conservation of the (renormalised) streamwise momentum flux. Inserting (5.1) into (5.6), we have

$$
\int_{-\infty}^{-\frac{y_{0}}{\delta}} \bar{U}_{B}\left(\eta^{\dagger}\right)\left[\bar{U}_{B}\left(\eta^{\dagger}\right)-\bar{U}_{-}\right] \mathrm{d} \eta^{\dagger}+\int_{-\frac{y_{0}}{\delta}}^{\infty} \bar{U}_{B}\left(\eta^{\dagger}\right)\left[\bar{U}_{B}\left(\eta^{\dagger}\right)-\bar{U}_{+}\right] \mathrm{d} \eta^{\dagger}=\frac{C_{0}}{\delta} .
$$

On taking a derivative of (5.7) with respect to $\tilde{x}$, the equation becomes

$$
\bar{U}_{B}\left(-y_{0} / \delta\right)\left(\bar{U}_{+}-\bar{U}_{-}\right)\left(\dot{y}_{0} \delta-y_{0} \dot{\delta}\right)=C_{0} \dot{\delta},
$$

which relates $\dot{y}_{0}$, the rate of drift of the shear-layer centre, to $\dot{\delta}$, the spreading rate of its thickness.

The corresponding transverse velocity $V_{B}$ can be found by introducing a stream function $\psi_{B}$ such that

$$
\bar{U}_{B}=\frac{\partial \psi_{B}}{\partial y}=\frac{1}{\delta} \frac{\partial \psi_{B}}{\partial \eta^{\dagger}}, \quad \bar{V}_{B}=-\frac{\partial \psi_{B}}{\partial \tilde{x}}
$$

Integration of the first equation yields

$$
\psi_{B}=\delta\left[\int_{-\infty}^{\eta^{\dagger}}\left(\bar{U}_{B}-\bar{U}_{-}\right) \mathrm{d} \eta^{\dagger}+\bar{U}_{-} \eta^{\dagger}\right]+d_{0}(\tilde{x})
$$

and so

$$
V_{B}=-\dot{\delta}\left[\int_{-\infty}^{\eta^{\dagger}}\left(\bar{U}_{B}-\bar{U}_{-}\right) \mathrm{d} \tilde{\eta}+\bar{U}_{-} \eta^{\dagger}\right]+\left(\dot{y}_{0}+\eta^{\dagger} \dot{\delta}\right) \bar{U}_{B}-\dot{d}_{0}(\tilde{x})
$$

where $d_{0}(\tilde{x})$ is an unknown function of $\tilde{x}$. In order to determine $d_{0}(\tilde{x})$, which affects nonlinear dynamics of the CS, we now analyse the impact of the viscous shear layer on the mean flow in the far field. From (5.10), one finds that

$$
V_{B} \rightarrow \bar{U}_{ \pm} \dot{y}_{0}(\tilde{x})-\dot{d}_{0}(\tilde{x}) \equiv \bar{V}_{ \pm} \text {as } y \rightarrow \pm \infty
$$

where $\bar{V}_{ \pm}$are the so-called 'transpiration velocities', i.e. the transverse velocities induced by the viscous motion in the shear layer. Through $V_{ \pm}$, an $O\left(R_{T}^{-1}\right)$ perturbation $R_{T}^{-1}\left(\hat{u}^{ \pm}, \hat{v}^{ \pm}, \hat{p}^{ \pm}\right)$is induced in the far field corresponding to $\tilde{y} \equiv y / R_{T}=O(1)$, where the scaled velocity $\left(\hat{u}^{ \pm}, \hat{v}^{ \pm}\right)$and pressure $\hat{p}^{ \pm}$are functions of $\tilde{x}$ and $\tilde{y}$. They satisfy the same equations as (4.14) provided that $\bar{x}$ and $\bar{y}$ are replaced by $\tilde{x}$ and $\tilde{y}$ respectively. It it follows that $\hat{p}^{ \pm}$satisfies the Laplace equation

$$
\nabla^{2} \hat{p}^{ \pm}=0,\left.\quad \frac{\partial \hat{p}^{ \pm}}{\partial \tilde{y}}\right|_{\tilde{y}=0}=-\bar{U}_{ \pm} \frac{\partial \bar{V}_{ \pm}}{\partial \tilde{x}} \quad\left(-x_{n}<\tilde{x}<\infty\right)
$$

where the boundary condition corresponds to the matching with the transpiration velocities at the upper and lower outer edges of the shear layer. The solution for $\hat{p}^{ \pm}$may depend on other bounding surfaces that may be present in an experimental setup. On the assumption that any asymmetry in the upper and lower streams is absent, then $\hat{p}^{ \pm}$are determined by the boundary conditions at $\tilde{y}=0$. A simple order-of-magnitude argument indicates that a mixing layer cannot sustain a pressure difference of $O\left(R_{T}^{-1}\right)$, which means that $p^{+}=p^{-}$on $\tilde{y}=0$. This requires $-\bar{U}_{+} \bar{V}_{+}=\bar{U}_{-} \bar{V}_{-}$, from which one finds

$$
\dot{d}_{0}=\frac{\bar{U}_{+}^{2}+\bar{U}_{-}^{2}}{\bar{U}_{+}+\bar{U}_{-}} \dot{y}_{0}(\tilde{x})
$$

The relations (5.7), (5.8) and (5.12) hold for any $\tilde{x}$. Specifically, putting $\tilde{x}=0$ where $y_{0}=0$ and $\delta=1$, we have

$$
\int_{-\infty}^{0} \bar{U}_{B}\left(\bar{U}_{B}-\bar{U}_{-}\right) d \eta^{\dagger}+\int_{0}^{\infty} \bar{U}_{B}\left(\bar{U}_{B}-\bar{U}_{+}\right) d \eta^{\dagger}=C_{0}
$$




$$
\dot{y}_{0}=\frac{C_{0} \dot{\delta}}{\bar{U}_{c}\left(\bar{U}_{+}-\bar{U}_{-}\right)}, \quad \dot{d}_{0}=\frac{\bar{U}_{+}^{2}+\bar{U}_{-}^{2}}{\bar{U}_{c}\left(\bar{U}_{+}^{2}-\bar{U}_{-}^{2}\right)}\left(C_{0} \dot{\delta}\right) .
$$

In our calculations, we choose the profile

$$
\bar{U}_{B}=\bar{U}_{c}+\left(1+q_{c} \operatorname{sech}^{2} \eta^{\dagger}\right) \tanh \eta^{\dagger},
$$

which GKW found to fit their low-excitation experimental data for $q_{c}=0.67$. The special case of $q_{c}=0$ has been the popular choice in theoretical studies as the neutral mode can be found analytically. Substitution of (5.15) into (5.13) and (5.4) gives the constants

$$
C \equiv C_{0} / 2=\ln 2-1+\frac{1}{6} q_{c}+\frac{2}{15} q_{c}^{2}, \quad C_{1}=\left(1-2 q_{c} / 3-2 q_{c}^{2} / 15\right) / 2 .
$$

It follows that

$$
\bar{U}_{c}^{\prime}=1+q_{c}, \quad \bar{U}_{c}^{\prime \prime \prime}=-2\left(1+4 q_{c}\right) ; \quad \bar{U}_{1, c}=-\left(1+q_{c}\right) C \dot{\delta} / \bar{U}_{c}, \quad \bar{U}_{1, c}^{\prime \prime}=2\left(1+4 q_{c}\right) C \dot{\delta} / \bar{U}_{c} .
$$

For the evaluation of $I_{1}$, only the odd parts of $\bar{U}_{1}$ and $\bar{U}_{1}^{\prime \prime}$ are required, which are found as

$$
\bar{U}_{1}=-\eta \bar{U}_{B}^{\prime} \dot{\delta}+\text { even part, } \quad \bar{U}_{1}^{\prime \prime}=-\eta \bar{U}_{B}^{\prime \prime \prime} \dot{\delta}+\text { even part. }
$$

Use of these in (3.17) shows that $I_{1}$ may be written as

$$
I_{1}=\hat{I}_{1} \dot{\delta}, \quad \hat{I}_{1}\left(q_{c}\right)=\int_{-\infty}^{\infty} \frac{(\bar{U}-c) \bar{U}^{\prime \prime \prime}-\bar{U}^{\prime \prime} \bar{U}^{\prime}}{(\bar{U}-c)^{2}} \eta \phi_{1}^{2} \mathrm{~d} \eta
$$

The parameters characterizing non-parallelism are found as

$$
\left.\begin{array}{c}
\chi=-\alpha\left(1+q_{c}\right)\left(\ln 2-\frac{1}{2} q_{c}+C+C / c^{2}\right) b_{s}, \quad \chi_{2}=0, \\
\Lambda_{0}=-\frac{\alpha c\left(1+q_{c}\right)^{2} \hat{I}_{1}\left(q_{c}\right)}{2\left(1+4 q_{c}\right)} b_{s}, \quad \sigma_{s}=\frac{\alpha c\left(1+q_{c}\right)^{2} \hat{I}_{1}}{2\left(1+4 q_{c}\right)\left(\pi+\Lambda_{2}\right)} b_{s} \equiv \sigma_{0} b_{s},
\end{array}\right\}
$$

where

$$
b_{s}=\frac{\mathrm{d} \theta^{*}}{\mathrm{~d} x^{*}} /\left(\epsilon C_{1}\right)
$$

and

$$
\Lambda_{2}=-\frac{\mathrm{i} \alpha^{2}\left(1+q_{c}\right)^{2} J_{1}\left(q_{c}\right)}{c\left(1+4 q_{c}\right)}
$$

When $q_{c}=0$, it is found that

$$
\phi_{1}=\operatorname{sech} \eta^{\dagger}, \quad \alpha=1, \quad c=\bar{U}_{c}, \quad J_{1}=2, \quad \hat{I}_{1}=-4, \quad J_{2}=0,
$$

but for $q_{c} \neq 0$, it is necessary to solve the Rayleigh equation numerically to find neutral mode, and then evaluate $\hat{I}_{1}$ and $J_{1}$; here $\hat{I}_{1}$ is a Cauchy principal value but can be computed easily using its definition.

\subsection{Numerical results}

Flow quantities of interest include the amplitude $A$ of the fundament component and the so-called 'critical-layer vorticity' $\Omega_{c}^{\dagger}$ of CS. The latter is defined as (see Goldstein \& Leib 1988)

$$
\Omega_{c}^{\dagger}=\frac{1}{2} \bar{U}_{c}^{\prime \prime \prime}+\left(\Psi_{2, Y Y}+\Psi_{0, \zeta \zeta}\right)=\frac{1}{2} \bar{U}_{c}^{\prime \prime \prime} Y^{2}+\Omega^{\dagger}+\left(\bar{U}_{c}^{\prime \prime \prime} / \bar{U}_{c}^{\prime}\right) A^{\dagger} \mathrm{e}^{\mathrm{i} \bar{\zeta}}+c . c .,
$$




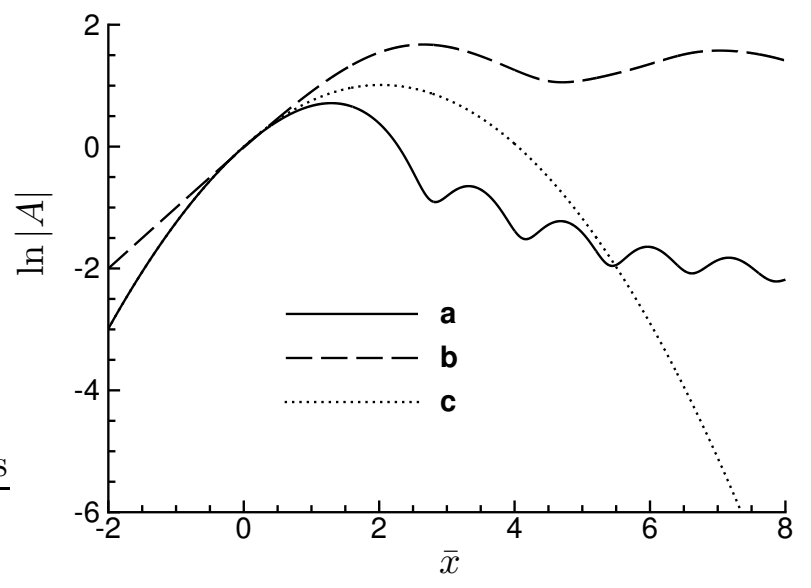

Figure 1: Nonlinear development of the amplitude $A$. Solid line (curve a): nonparallelnonlinear theory with $\mathrm{d} \theta^{*} / \mathrm{d} x^{*}=1 / 54$; dashed line (curve $\mathrm{b}$ ): parallel-nonlinear theory $\mathrm{d} \theta^{*} / \mathrm{d} x^{*}=0$; dotted line (curve c): nonparallel-linear theory.

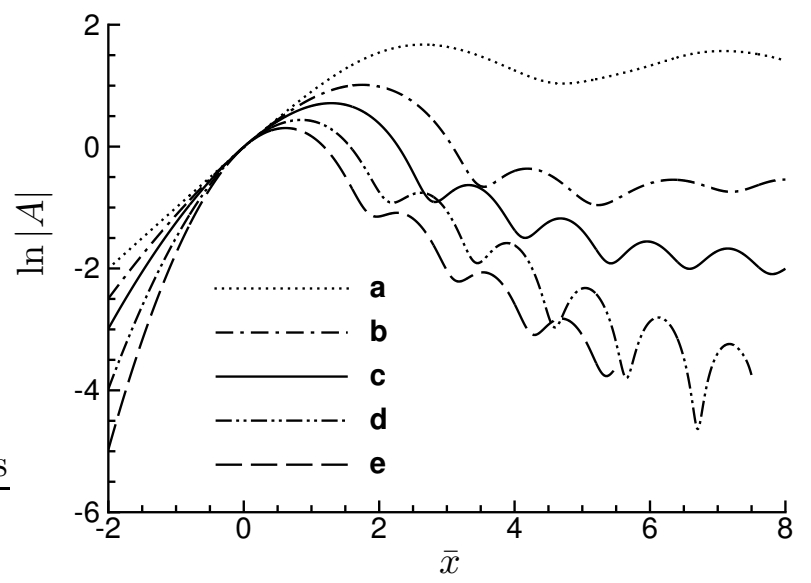

Figure 2: Nonlinear development of the amplitude $A$ for different spread rates of the momentum thickness $\mathrm{d} \theta^{*} / \mathrm{d} x^{*}=0$ (curve a), 1/108 (curve b), $1 / 54$ (curve c), $1 / 27$ (curve d) and $1 / 18$ (curve e). 
with which $\left(\bar{U}_{c}^{\prime}+\epsilon \Omega_{c}^{\dagger}\right)$ represents the total vorticity within the critical layer. For convenience, the results will be presented by plotting the renormalized vorticity

$$
\Omega_{c} \equiv \Omega_{c}^{\dagger}\left(\alpha \bar{U}_{c}^{\prime}\right)^{2} / \bar{U}_{c}^{\prime \prime \prime}=\frac{1}{2}\left(\eta+S_{0}\right)^{2}+A \mathrm{e}^{\mathrm{i} \bar{\zeta}}+\text { c.c. }+\Omega .
$$

The choice of the parameters is guided by the experiments of GKW, where $U_{1}^{*}=10 \mathrm{~m} / \mathrm{s}$, $U_{2}=4 \mathrm{~m} / \mathrm{s}, \mathrm{d} \theta^{*} / \mathrm{d} x^{*}=1 / 54$ (see the data set III in Table 1 of GKW) and the momentum thickness $\theta^{*}=18.78 \mathrm{~mm}$, which can be read from figure 1 of GKW. For the purpose of demonstrating qualitative behaviours of the evolution, we take $q_{c}=0$, for which $\delta_{0}^{*}=$ $\theta^{*} / C_{1}$ with $C_{1}=0.5$. Based on $\delta_{0}^{*}$ and $U_{0}^{*}$, the Reynolds number $R$ turns out to be 7100 . We choose $S_{0}<0$ such that $\kappa_{r}=1$, and set the amplitude $\epsilon=0.1036$, for which nonlinear effects are significant, and the parameters characterizing the non-parallel-flow effects, $\chi$ and $\sigma_{s}$, take $O(1)$ values: $\chi=-0.1180$ and $\sigma_{s}=-0.4943-0.1349 \mathrm{i}$. The eddy viscosity and phase lag have to be treated as free parameters because no experimental data about them are available. The expression (3.40) implies that if $\hat{\tau} \neq 0$ and $\bar{\lambda}_{2}>\bar{\lambda}_{1}$ (i.e. $\tilde{R}_{T}<R$ ), the effective diffusion coefficient $\bar{\lambda}_{1}+\bar{\lambda}_{2} \cos (n \hat{\theta})(\hat{\theta} \equiv \alpha c \hat{\tau})$ will become negative for certain $n$, rendering the problem ill-posed. This unfavourable feature reflects a shortcoming of the model, which assumes the same relaxation time $\hat{\tau}$ for the fundamental and all harmonics. Improved models are being pursued, and here the ill-poseness is avoided by restricting $\tilde{R}_{T} \leq R$, i.e. $\bar{\lambda}_{2} \leq \bar{\lambda}_{1}$. Specifically, we choose $\tilde{R}_{T}=R$, for which $\bar{\lambda}_{2}=\bar{\lambda}_{1}=0.00422$, and take phase lag $\hat{\theta}=\pi / 5$. These values are rather arbitrary. Fortunately, the evolution of the CS turns out to be rather insensitive to either of $\hat{\theta}$ and $\bar{\lambda}_{2}$ as will be shown later.

Figure 1 shows the amplitude evolution (curve a) under the influence of both nonlinearity and non-parallelism. In order to see their role, the result when either of these being artificially suppressed is also plotted. Without nonlinearity, the development is Gaussian, featuring a broad peak and rapid monotonic decay (curve c). On the other hand, with the nonlinear effect included but non-parallelism neglected (curve b), the amplitude undergoes oscillatory saturation (and actually slight algebraic amplification; see later). Simultaneous effects of non-parallelism and nonlinearity render the amplitude to decay in an oscillatory manner, which is obviously different from the linear approximation and parallel-nonlinear theory. The neutral position of the mode with frequency $\omega_{n}=\alpha c$ corresponds to $\bar{x}=0$, but the disturbance under consideration has the frequency $\alpha c+\epsilon^{1 / 2} S_{0}<\alpha c \equiv \omega_{n}$, and so it becomes linearly neutral and attains its maximum somewhat downstream of $\bar{x}=0$.

In the present theory, non-parallelism manifests in two ways: (a) it alters the growth rate of the CS, which is well known, and (b) induces a translating critical layer, the effect of which becomes active in the nonlinear stage of the evolution. Non-parallelism is controlled by the spreading rate of the shear-layer thickness $\mathrm{d} \theta^{*} / \mathrm{d} x^{*}$. The amplitude evolution for a range of $0 \leq \mathrm{d} \theta^{*} / \mathrm{d} x^{*} \leq 1 / 18$ is displayed in figure 2. As is illustrated, non-parallelism tends to inhibit CS in the sense that the decay starts earlier and becomes faster, and the peak value decreases as $\mathrm{d} \theta^{*} / \mathrm{d} x^{*}$ increases. However, the overall feature of oscillatory attenuation remains. Such oscillatory attenuations have been observed in a number of experiments (Fiedler \& Mensing 1985, WW, Hussain \& Thompson 1980, Zaman \& Hussain 1980). It is worth noting that the amplitude $\epsilon$ influences the evolution through $\chi, \Lambda_{0}, \sigma_{s}, \bar{\lambda}_{1}$ and $\bar{\lambda}_{2}$. Since the first three of these parameters are proportional to $b_{s}=\mathrm{d} \theta^{*} / \mathrm{d} x^{*} /\left(C_{1} \epsilon\right)$ (see (5.18)) while $\bar{\lambda}_{1}$ and $\bar{\lambda}_{2}$ are practically zero, curves $\mathrm{b}$ and $\mathrm{d}$ would represent approximately the evolution for $\epsilon=0.2072$ (doubled) and 0.0518 (halved) respectively with a fixed $\mathrm{d} \theta^{*} / \mathrm{d} x^{*}=1 / 54$.

We now exam the role of molecular and eddy viscosities, represented by $\bar{\lambda}_{1}$ and $\bar{\lambda}_{2}$ respectively. The amplitude evolution for different values of these parameters is shown in figure 3, where the corresponding result with non-parallelism being ignored is also 


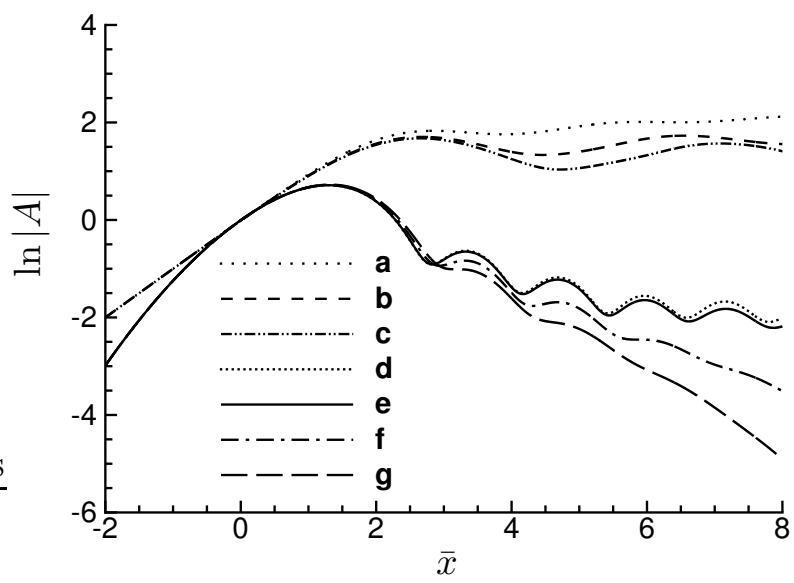

Figure 3: The development of the amplitude $A$ for different viscosity parameters $\bar{\lambda}_{1}$ and $\bar{\lambda}_{2}$. Parallel-nonlinear theory $\left(\mathrm{d} \theta^{*} / \mathrm{d} x^{*}=0\right)$ for $\bar{\lambda}_{1}=\bar{\lambda}_{2}=0.5$ (curve a), 0.1 (curve b), 0 (curve c). Nonparallel-nonlinear theory $\left(\mathrm{d} \theta^{*} / \mathrm{d} x^{*}=1 / 54\right)$ for $\bar{\lambda}_{1}=\bar{\lambda}_{2}=0$ (curve $\mathrm{d}$ ), 0.0442 (curve e), 0.05 (curve f) and 0.1 (curve g).
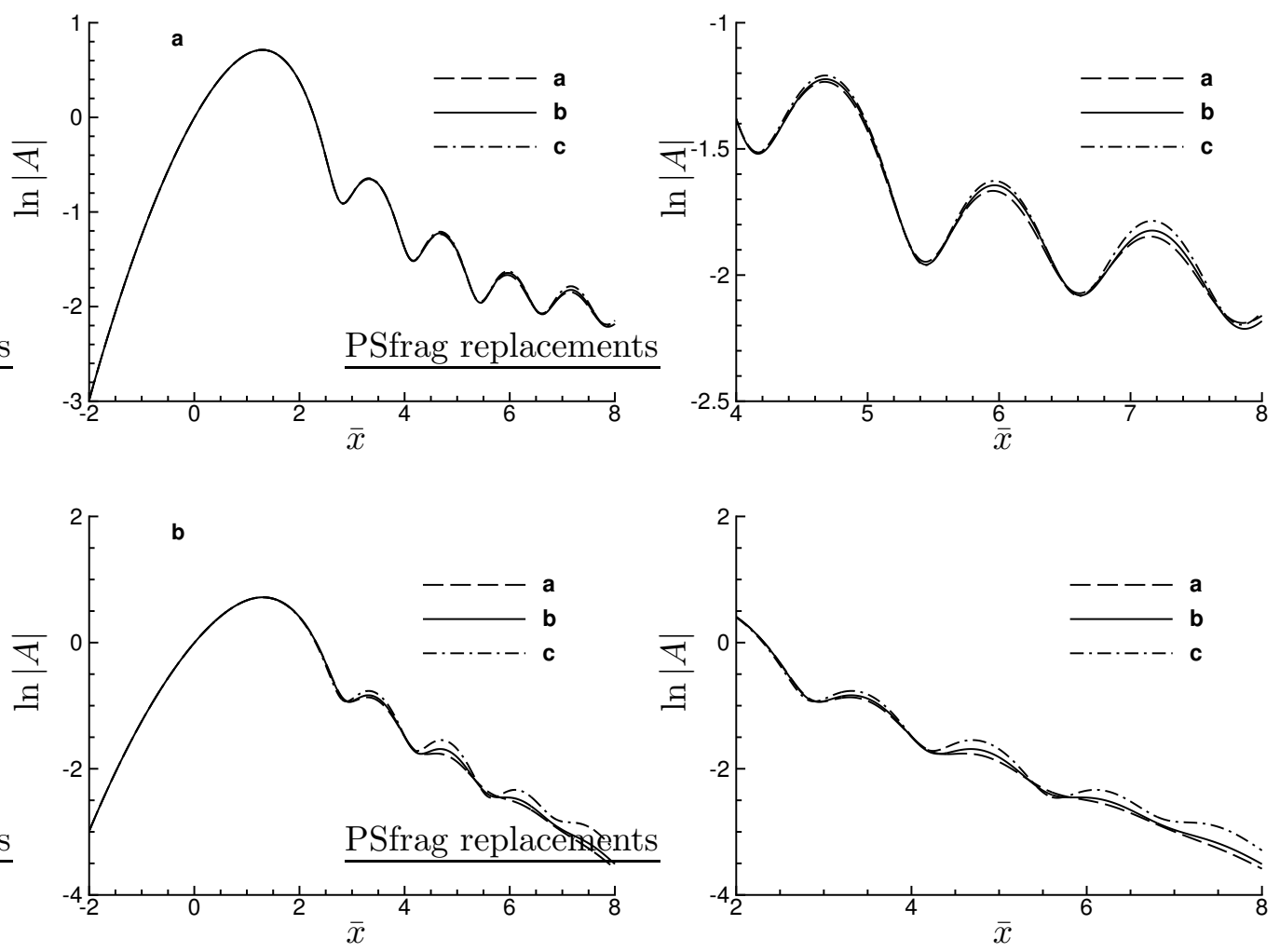

Figure 4: The development of the amplitude $A$ for different phase lag $\hat{\theta}=\alpha c \hat{\tau}$. The top row is for $\bar{\lambda}_{1}=\bar{\lambda}_{2}=0.00422$ and $\hat{\theta}=0$ (curve a), $\pi / 5$ (curve b) and $\pi / 2$ (curve c); the right figure is an enlarged view for $4<\bar{x}<8$. The bottom row is for $\bar{\lambda}_{1}=\bar{\lambda}_{2}=0.05$ and $\hat{\theta}=0$ (curve a), $\pi / 5$ (curve b) and $\pi / 2$ (curve c); the right figure is an enlarged view for $2<\bar{x}<8$. 


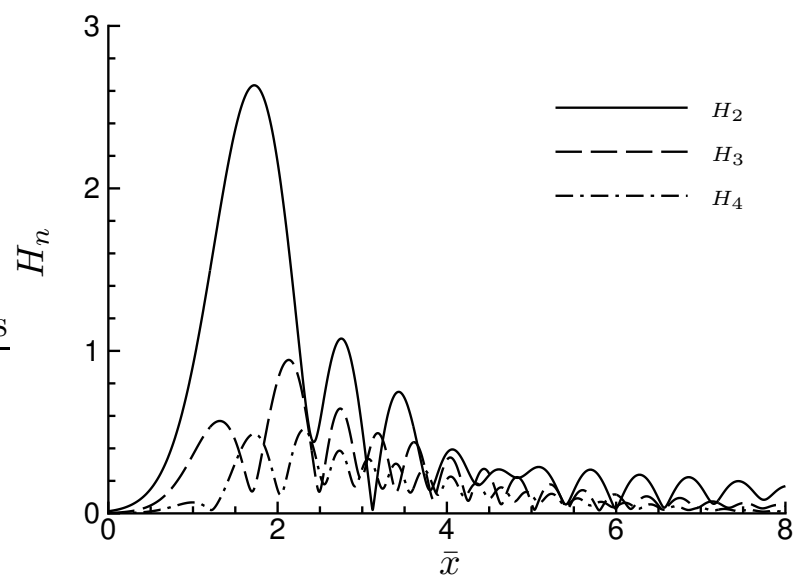

Figure 5: The development of the harmonics as shown by $H_{n} \equiv a_{2}^{(n)+}-a_{2}^{(n)-}(n=2,3,4)$ for $\mathrm{d} \theta^{*} / \mathrm{d} x^{*}=1 / 54, \bar{\lambda}_{1}=\bar{\lambda}_{2}=0.0422$ and $\hat{\theta}=\pi / 5$.

presented. Neither of the viscosities affects the linear stage of the development, and their impact on the nonlinear evolution is opposite depending on if non-parallelism is included. When non-parallelism is neglected, the amplitude continues to amplify and eventually follows an algebraic growth (Goldstein \& Hutlgren 1988). The amplitude is greater for larger values of $\bar{\lambda}_{1}$ and $\bar{\lambda}_{2}$, indicating that viscosities promote the growth of the perturbation, the reason being that nonlinearity plays a stabilizing role and a larger viscosity corresponds to weaker nonlinearity and therefore a less stabilizing effect. On the contrary, when non-parallelism is included, increasing viscosities tends to enhance decay and reduce the oscillation as is expected intuitively. The quantitative difference indicates the importance of accounting for non-parallelism. In comparison, viscous effects are rather weak, affecting only the later stage of the development since typical values of $\bar{\lambda}_{1}$ and $\bar{\lambda}_{2}$ turn out to be fairly small. This is entirely consistent with the assertion made by Fiedler \& Mensing (1985) based on their experimental observations.

The effect of the phase lag $\hat{\theta} \equiv \alpha c \hat{\tau}$ is also considered. Figure 4 shows the amplitude evolution for different $\hat{\theta}$ with typical values of $\bar{\lambda}_{1}=\bar{\lambda}_{2}=0.004$ as well as with artificially elevated values, $\bar{\lambda}_{1}=\bar{\lambda}_{2}=0.05$. In both cases, increasing $\hat{\theta}$ only slightly alleviates the decay, which may be attributed to a slightly smaller total viscosity $\left(\bar{\lambda}_{1}+\bar{\lambda}_{2} \cos \hat{\theta}\right)$. The results in figures 3 and 4 indicate that the phase lag and the magnitude of the eddy viscosity have a negligible influence on the CS, suggesting that the latter evolves practically in the same manner as an instability wave on a laminar shear layer. Indeed, the dynamics is essentially inviscid since the effect of molecular viscosity is more likely to be even smaller and negligible as well.

As was mentioned earlier, the most significant feature of the strongly nonlinear criticallayer theory is that all harmonics appear simultaneously at the same order. They are measured by the streamwise velocity jump

$$
H_{n} \equiv a_{2}^{(n)+}-a_{2}^{(n)-} .
$$

Figure 5 shows the development of $H_{n}$ for $n=2,3,4$. The size of $H_{n}$ decreases with $n$. In the earlier nonlinear phase, the first harmonic $H_{2}$ is dominant, but all three become more-or-less comparable in the later stage $2.2<\bar{x}<5$.

Simultaneous appearance of harmonics leads to roll-up of vortices. This process is illustrated in figure 6 by plotting contours of the normalized critical-layer vorticity of the CS at representative streamwise locations. At the same time distributions of the 

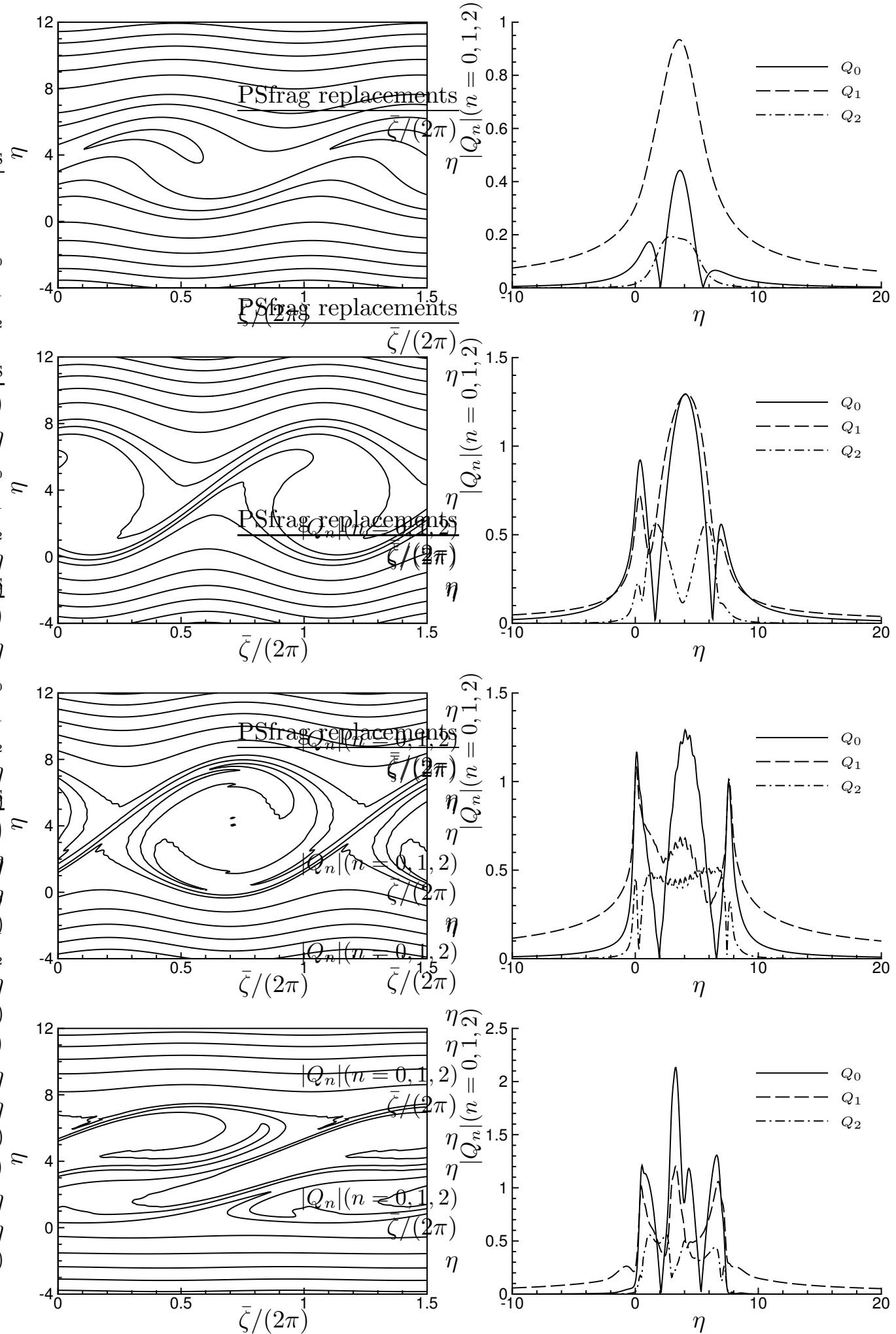

Figure 6: Contours of the critical-layer vorticity $\Omega_{c}$ (left column), and profiles of the fundamental $Q_{1}$, first harmonic $Q_{2}$ and the mean-distortion $Q_{0}$ (right column) at $\bar{x}=0$, $\bar{x}=1, \bar{x}=2$ and $\bar{x}=4$. 
fundamental $Q_{1}$, first harmonic $Q_{2}$ and the mean-flow distortion $Q_{0}$ are monitored. In the initial linear stage $\bar{x}=0$, the fundamental is dominant and the vorticity exhibits a simple pattern of 'cat's eye' associated with a simple sinusoidal perturbation. By $\bar{x}=1$, vorticity lines begin to fold and a vortex roller starts forming. The first harmonic acquires an amplitude about half of that of the fundamental, whereas the mean-flow distortion becomes comparable with the latter. The harmonic and the mean-flow distortion remain trapped in the critical layer as they decay rapidly towards its outer edges. When $\bar{x}=2$, a fully developed vortex roller has emerged. The relative magnitudes of the three components remain similar as at $\bar{x}=1$. However the profile of the first harmonic exhibits rapid oscillations. A further calculation with the mesh size halved indicates that they are well resolved. At $\bar{x}=4$, vortices become elongated in the streamwise direction and there is considerable overlapping between two adjacent vortices. This might be related to "partial pairing' observed in experiments (Hussain \& Clarke 1981). In this case, vortices appear to merge without involving the subharmonic.

The Reynolds stress and the mean-flow distortion are calculated. For the chosen profile (5.15) with $\left(q_{c}=0\right)$, it is found that

$$
\left.\begin{array}{c}
\mathcal{F}_{1}+\hat{C}^{ \pm} \mathcal{F}_{0}=\frac{1}{2}[\tanh y \sinh y \mp(y \operatorname{sech} y+\sinh y)], \\
\mathcal{F}_{2}+\hat{C}^{ \pm} \mathcal{F}_{0}=-\left[\sinh y \ln |\tanh y|+\operatorname{sech} y \int_{0}^{y} \ln |\tanh s| \mathrm{d} s\right], \\
\hat{S}=\operatorname{sech}^{4} y\left(1+2 \bar{U}_{c} \operatorname{coth} y\right), \\
I_{s}^{+}-I_{s}^{-}=\frac{2}{\bar{U}_{c}} \ln \left|\frac{U_{c}+1}{U_{c}-1}\right| .
\end{array}\right\}
$$

Substitution of (5.22) into (4.6) gives

$$
\begin{aligned}
\tau_{12}= & -\frac{\partial|A|^{2}}{\partial \bar{x}}\left\{\operatorname{sech}^{2} y+2 \ln |\tanh y|+c^{-1}\left[\left(2+\operatorname{sech}^{2} y\right) \tanh y \mp 2\right]\right\} \\
& -\left[\mathrm{i} A^{*} \operatorname{sech} y\left(U_{3 / 2}-\mathrm{i}(-1 \pm 2 / c) A_{\bar{x}}+2 \mathrm{i} A_{\bar{x}} \ln |\eta|\right)+c . c .\right]
\end{aligned}
$$

where the jump in the outer solution is smoothed out by the contribution of the inner solution $U_{3 / 2}$.

Substituting (5.23) and (5.24) into (4.26), we find that for $\Delta \Theta$,

$$
\begin{aligned}
\Delta \Theta= & \epsilon^{2}|A|^{2}\left\{\frac{1}{3}-\frac{1}{\bar{U}_{c}^{2}}+\frac{\bar{U}_{c}^{2}-1}{2 \bar{U}_{c}} \ln \left|\frac{\bar{U}_{c}+1}{\bar{U}_{c}-1}\right|\right\} \\
& -\frac{1}{2} \epsilon^{2} \int_{-\infty}^{\infty} \tanh y\left\{-\frac{\bar{U}_{c}^{\prime \prime \prime} / \bar{U}_{c}^{\prime 2}}{y-y_{c}}|A|^{2}+\epsilon^{-1 / 2} U_{M}^{(1)}\right\} \mathrm{d} y .
\end{aligned}
$$

The result reduces to that of Goldstein \& Leib (1988) when the contribution from the critical layer is ignored. Finally the indefinite integral in (4.13) can be evaluated analytically. Inserting the result along with (5.23) into (4.23), we obtain the streamwise velocity of the mean-flow distortion,

$$
\begin{aligned}
U_{M}= & \epsilon^{3 / 2} U_{M}^{(1)}+\epsilon^{2}|A|^{2}\left\{\operatorname{sech}^{2} y\left[a_{3}^{(0) \pm}+\frac{2}{\bar{U}_{c}} \ln \left|\frac{\bar{U}_{c}+\tanh y}{\left(\bar{U}_{c} \pm 1\right) \tanh y}\right|\right]-2 \operatorname{sech}^{4} y \operatorname{coth} y+\frac{2}{y-y_{c}}\right\} \\
& +\epsilon^{2}\left(U_{M}^{(2)}+\frac{2}{\bar{U}_{c}}|A|^{2} \ln |\eta|\right) .
\end{aligned}
$$



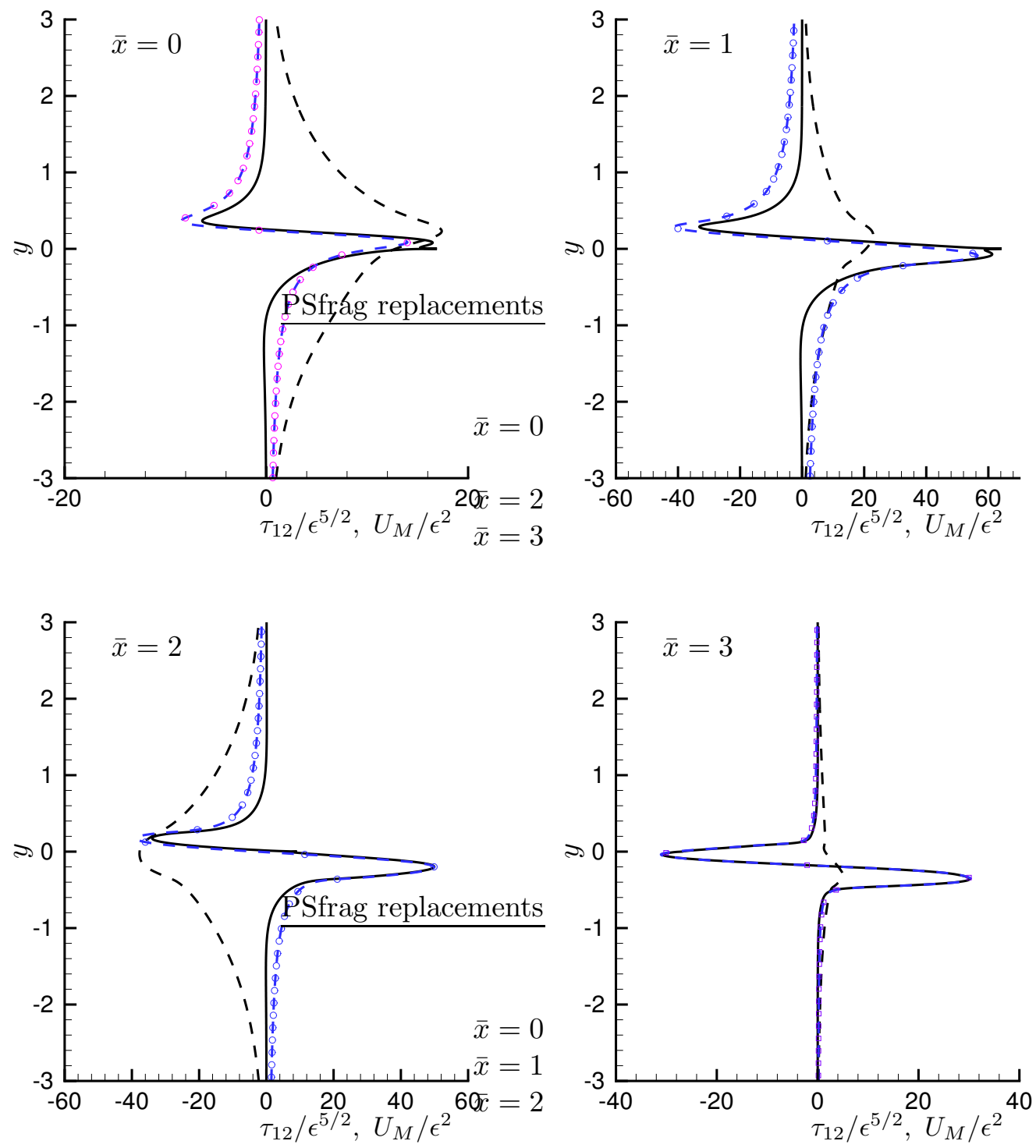

Figure 7: Distribution of the CS-induced Reynolds stress $\tau_{12}$ (dashed lines) and mean-flow distortion $U_{M}$ (solid lines) at $\bar{x}=0,1,2$ and 3. A small, $O\left(\epsilon^{2}\right)$, discontinuity at $y=y_{c}=0$ arises due to neglecting $\left(U_{M}^{(2)}-c_{M}|A|^{2} \ln |\eta|\right)$. The dashed lines with symbols denote $U_{M}^{(1)}$, the leading-order inner solution. 


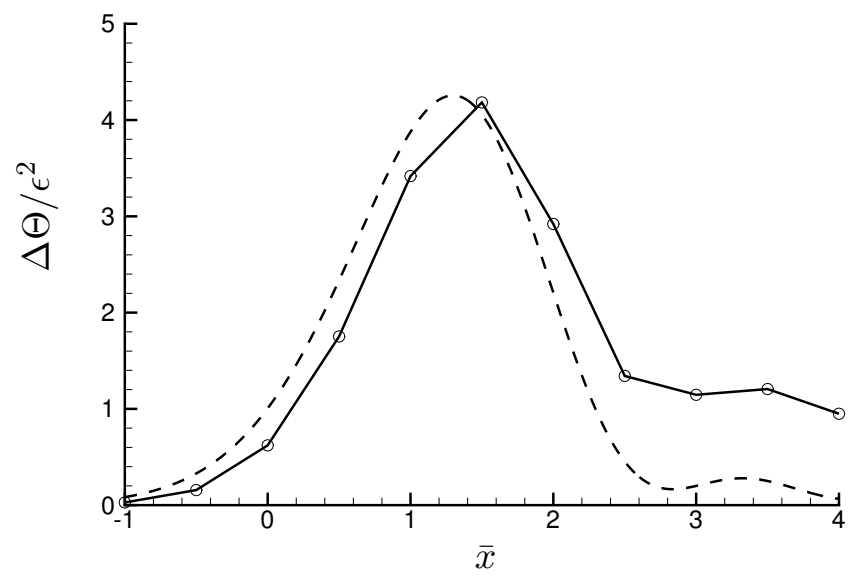

Figure 8: The change of the momentum thickness $\Delta \Theta / \epsilon^{2}$ versus $\bar{x}$. Solid line: full result calculated using (5.26); dashed line: the result with the contribution from the critical layer being omitted.

As was mentioned earlier, equation (4.27) coupled with an amplitude equation for $B^{\dagger}$ has to be solved to find $U_{M}^{(2)}$. As the calculation is highly complex, we shall ignore $\left(U_{M}^{(2)}+2 / U_{c}|A|^{2} \ln |\eta|\right)$, which delays like $1 / \eta$ as $\eta \rightarrow \infty$. The resultant $O\left(\epsilon^{2}\right)$ error causes a small discontinuity at $y=y_{c}$, but does not change the overall feature of the solution. In calculations, use is made of the relation

$$
y=\epsilon^{1 / 2}\left(\eta+S_{0}\right)+\frac{C \mathrm{~d} \theta^{*} / \mathrm{d} x^{*}}{C_{1} \epsilon^{1 / 2}} \bar{x} .
$$

Figure 7 displays the distributions of the Reynolds stress $\tau_{12}$ and the mean-flow distortion $U_{M}$ at four representative streamwise locations. At $\bar{x}=0$ and 1 , the disturbance is amplifying, and the Reynolds stress $\tau_{12}>0$ almost everywhere. The amplitude of the CS peaks at $\bar{x} \approx 1.3$, downstream of which (e.g. at $\bar{x}=2$ ) $\tau_{12}<0$ for all $y$, giving rise to a negative production. As a result, the energy is transferred from the perturbation to the mean flow in this region. At $\bar{x}=3, \tau_{12}$ becomes positive again, but its value has almost vanished. Overall feature of the mean-flow distortion, which concentrates in the critical layer, is well represented by the leading-order inner solution $U_{M}^{(1)}$. Interestingly, the meanflow distortion still persists and retains a significant magnitude farther downstream due to the non-equilibrium effect. That the Reynolds stress changes its sign twice is consistent with the experimental observations shown in figures 8(a) and 15 of WW.

Figure 8 shows the change of the momentum thickness. That $\Delta \Theta>0$ at all locations indicates that the shear layer thickens in the presence of CS, consistent with experimental observations. Before the peak position, the contribution from the critical layer is small but appreciable. Its effect becomes rather significant in the attenuating phase as the contribution from outside of the critical layer vanishes quickly. The slow relaxation of the mean-flow distortion and the shear-layer thickness implies that the imprint of the CS in these two related quantities lasts longer than the CS itself as has been observed in experiments (e.g. Wygnanski et al. 1986). This long memory must be associated with the non-equilibrium effect in the critical layer, which causes the mean-flow distortion to be history dependent, whereas the distortion outside is proportional to $|A|^{2}$ (i.e. memoryless) as (4.13) indicates. 


\subsection{Comparison with experiments}

Let the dimensional variables/quantities be indicated by the superscript $*$. In order to facilitate the comparison with experiments, note that there exist the following relationships with the non-dimensional variable/quanties adapted in the present paper,

$$
\bar{x}=\epsilon^{1 / 2} c^{-1} x=\epsilon^{1 / 2} c^{-1} C_{1}\left(x^{*}-x_{n}^{*}\right) / \theta^{*}, \quad y=C_{1} y^{*} / \theta^{*}, \quad v=2 v^{*} /\left(U_{1}^{*}-U_{2}^{*}\right),
$$

where $C_{1}$ is a profile-dependent constant defined by (5.4). The dimensional frequency $f^{*}$ is related to $\omega \equiv \alpha c$ (with $S_{0}=0$ ) by the relation

$$
\alpha c=\frac{4 \pi f^{*} \theta^{*}}{C_{1}\left(U_{1}^{*}-U_{2}^{*}\right)}
$$

In the experiment of WW, the measured amplitude of CS, shown in figure 8(b) of their paper, is represented by the integrated intensity

$$
A_{\exp }=\frac{f^{*} \int_{-\infty}^{\infty}\left|<v^{*}>\right| \mathrm{d} y^{*}}{\left(U_{1}^{*}-U_{2}^{*}\right) U_{c}^{*}}
$$

where $U_{c}^{*}=\left(U_{1}^{*}+U_{2}^{*}\right) / 2$ and $\langle\cdot>$ represents the amplitude of the time-dependent signature. Application of the relations in (5.28) to (5.30) yields

$$
\int_{-\infty}^{\infty}|<v>| \mathrm{d} y=\frac{C_{1}\left(U_{1}^{*}+U_{2}^{*}\right)}{f^{*} \theta^{*}} A_{\text {exp }}
$$

In the present work,

$$
v=-\epsilon \mathrm{i} \alpha A^{\dagger} \phi_{1}(y) \mathrm{e}^{\mathrm{i} \alpha(x-c t)}+c . c .=2 \epsilon\left(\alpha \bar{U}_{c}^{\prime}\right)^{-1} A \phi_{1}(y) \sin (\alpha x-\omega t) .
$$

It follows from (5.31) and (5.32) that

$$
\epsilon|A|=\frac{\alpha \bar{U}_{c}^{\prime}}{2 I_{v}} \frac{C_{1}\left(U_{1}^{*}+U_{2}^{*}\right)}{f^{*} \theta^{*}} A_{\mathrm{exp}},
$$

where

$$
I_{v}=\int_{-\infty}^{\infty}\left|\phi_{1}\right| d y
$$

In the linear regime, the amplitude function has the solution

$$
A=\exp \left\{\frac{1}{2} \sigma_{s} \bar{x}^{2}+\kappa \bar{x}\right\}=\exp \left\{\frac{1}{2} \sigma_{s}^{*}\left(x^{*}-x_{n}^{*}\right)^{2}+\kappa^{*}\left(x^{*}-x_{n}^{*}\right)\right\}
$$

where

$$
\sigma_{s}^{*}=\frac{\epsilon C_{1}^{2}}{\left(c \theta^{*}\right)^{2}} \sigma_{s}, \quad \kappa^{*}=\frac{C_{1} \epsilon^{1 / 2}}{c \theta^{*}} \kappa
$$

The parameter $\epsilon$ is determined by using (5.33), i.e. by requiring the predicted amplitude to be the same as the measured, at a suitable location $x^{*}$, e.g. at the peak position or in the linear regime upstream. For the latter case, the expression (5.34) may be used.

For the profile (5.15) with $q_{c} \neq 0$, the Rayleigh equation is solved numerically to find the neutral mode and to evaluate the constants related to the eigenfunction. The results for several $q_{c}$ are listed and contrasted with those for $q_{c}=0$ in Table 1.

The experiment of WW was performed for $U_{1}^{*}=10 \mathrm{~m} / \mathrm{s}, U_{2}^{*}=6 \mathrm{~m} / \mathrm{s}$ and $f^{*}=44.5 \mathrm{~Hz}$. Assuming that the profile is approximated by (5.15), we need to provide three parameters characterising the unperturbed mean flow: $q_{c}, \mathrm{~d} \theta^{*} / \mathrm{d} x^{*}$ and $\theta^{*}$. Unfortunately, they are 


\begin{tabular}{ccccccc}
\hline$q_{c}$ & $C$ & $C_{1}$ & $\alpha$ & $I_{v}$ & $J_{1}$ & $\hat{I}_{1}$ \\
\hline 0 & -0.3069 & 0.5 & 1 & $\pi$ & 2 & -4 \\
\hline 0.1 & -0.28885 & 0.4660 & 1.1828 & 2.6654 & 1.6954 & -4.4845 \\
\hline 0.3 & -0.2448 & 0.3940 & 1.5219 & 2.1278 & 1.3657 & -5.2233 \\
\hline 0.67 & -0.1353 & 0.2467 & 2.0665 & 1.6703 & 1.0920 & -6.0890 \\
\hline 0.9 & -0.04885 & 0.1460 & 2.3636 & 1.5156 & 0.9994 & -6.4316 \\
\hline
\end{tabular}

Table 1: Neutral wavenumber and the eigenfunction-related constants for typical $q_{c}$.

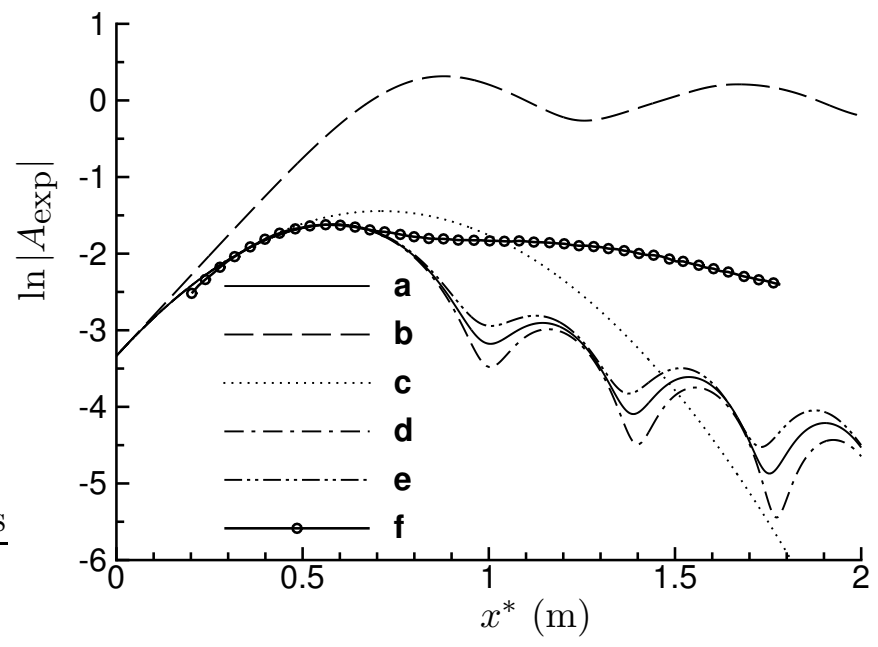

Figure 9: Comparison with the experimental data of WW. Curves (a)-(e) represent theoretical results for $q_{c}=0.9$ : (a) $\chi=-0.0306$, the value calculated for the experimental condition, (b) parallel-nonlinear theory, (c) linear solution including non-parallelism, (d) $\chi=-0.0612$, twice the experimental value, (e) $\chi=0$. Curve (f): experimental data. 


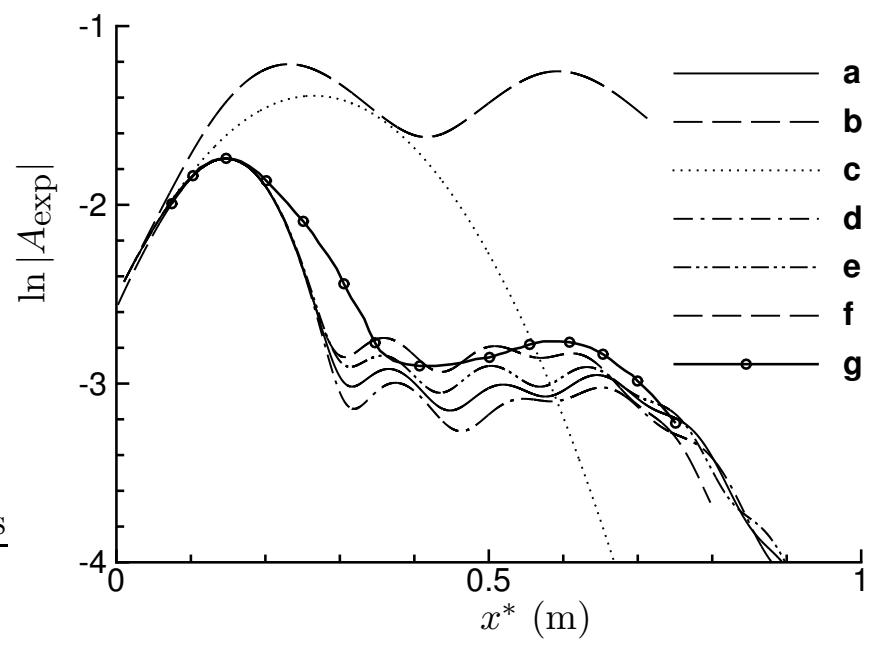

Figure 10: Comparison with the experimental data of Fiedler \& Mensing (1985). Curves (a)-(e) are for $q_{c}=0.1$ : (a) $\chi=-0.01729$, the value calculated for the experimental condition, (b) parallel-nonlinear theory, (c) linear solution including non-parallelism, (d) $\chi=-0.03458$, twice the experimental value, (e) $\chi=0$. Curve (f): $q_{c}=0.3$ and curve (g): experimental data.
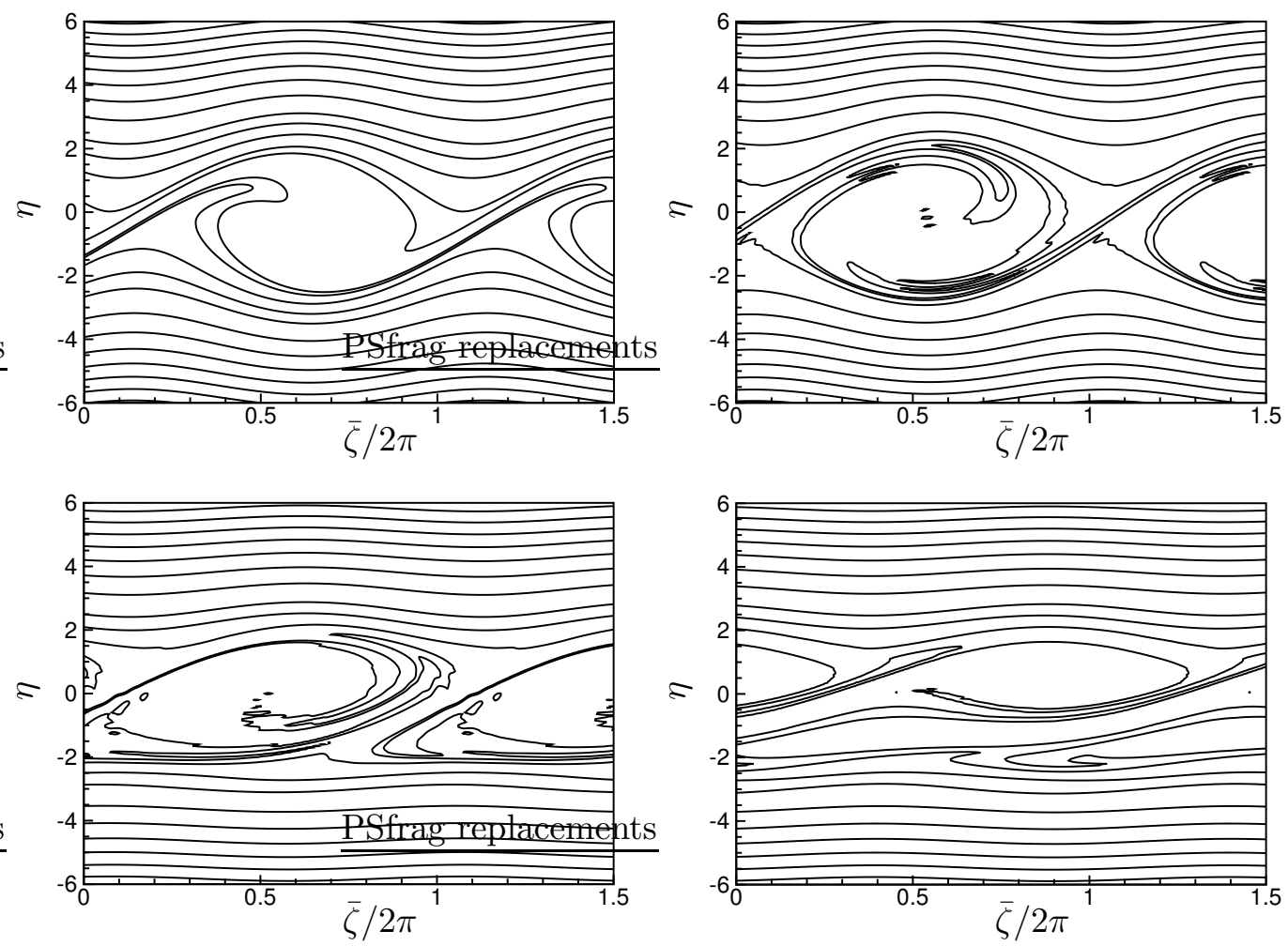

Figure 11: Vortex roll-up pertaining to the experimental condition of Fiedler \& Mensing (1985), illustrated by contours of $\Omega_{c}$, the normalised critical-layer vorticity of CS, at $x^{*}=0.1,0.2,0.3$ and $0.5 \mathrm{~m}$ from the leading edge. 
not available from the data of WW. Noting that the experiments of WW and GKW were performed in the same apparatus, we decide to estimate these parameters by appealing to the latter. Curiously, for $q_{c}=0.67$ the frequency of the neutral mode in GKW does not satisfy the constraint (5.29) despite that the profile was in agreement with the measurement. Our calculation shows that if $q_{c}=0.9$ is taken instead, the constraint is satisfied in all three cases of GKW. For the same experimental setup, the spreading rate of the unperturbed flow depends primarily on $U_{1}^{*} / U_{2}^{*}$ (Oster \& Wygnanski 1982, Weisbrot et al. 1982). This ratio in WW is the same as in the Case I of GKW, where $\mathrm{d} \theta^{*} / \mathrm{d} x^{*}=1 / 85$ was found. For controlled disturbance with $f^{*}=20 \mathrm{~Hz}$ and $U_{1}^{*}=5 \mathrm{~m} / \mathrm{s}$ and $U_{2}=3 \mathrm{~m} / \mathrm{s}$, the measured momentum thickness is about $11.5 \mathrm{~mm}$, which converts to a corresponding $\theta^{*} \approx 10 \mathrm{~mm}$ in WW by using (5.29), which indicates that the non-dimensional frequency $f^{*} \theta^{*} /\left(U_{1}^{*}-U_{2}^{*}\right)$ must be equal in the two experiments. Therefore, we take $q_{c}=0.9, \mathrm{~d} \theta^{*} / \mathrm{d} x^{*}=1 / 85$ and $\theta^{*}=10 \mathrm{~mm}$ in our calculations.

The amplitude development predicted by the theory is shown in figure 9 and compared with the experimental data of WW. The agreement is good in the linear and earlier nonlinear stages up to the peak position. Further downstream, the theory mimics the general trend, e.g. both the theoretical result and the measurement feature a local maximum downstream, but considerable discrepancy emerges: the decay predicted by the theory is much faster and the maximum is more pronounced than those measured. As $V_{B}$ and hence $\chi$ may be influenced by the detailed arrangements of the experiments, the value of $\chi$ is doubled, halved (not shown) and set to zero. None of these leads to significant change to the overall feature of the solution. However, if the non-parallel-flow effect is suppressed, the result (represented by the dashed line) bears little resemblance to the measurement. Even in the linear stage, reasonable agreement comes only when non-parallelism is accounted for. However, purely linear but nonparallel theory does not capture the characteristics of attenuation.

Calculations were also performed pertaining to the experiment of Fiedler \& Mensing (1985), where the free-stream velocities are $U_{1}^{*}=11 \mathrm{~m} / \mathrm{s}$ and $U_{2}^{*}=0$, and a disturbance with frequency $f^{*}=30 \mathrm{~Hz}$ was introduced through controlled excitation. Fiedler \& Mensing provided the spreading rate and thickness of the mixing layer for what they referred to as 'neutral flow', i.e. the state without artificial excitation. The mean flow is thus primarily influenced by small-scale turbulence and its spreading rate, $\mathrm{d} \theta^{*} / \mathrm{d} x^{*}=0.0354$, is used in our calculations. The experimental data for two weakest excitations, shown in figure 13 of their paper, indicate that the amplitude of the fundamental component saturates at $x^{*}$ where $S_{x}=f^{*}\left(x^{*}-x_{0}^{*}\right) / U_{1}^{*} \approx 1.25\left(x_{0}^{*} \approx-88 \mathrm{~mm}\right)$, which corresponds to $x^{*} \approx 370 \mathrm{~mm}$ and $\theta^{*} \approx 16 \mathrm{~mm}$ as can be inferred from figure 28 of Fiedler \& Mensing (1985). This position is taken as an approximation for the neutral location of the imposed disturbance, and hence the value $\theta^{*}=16 \mathrm{~mm}$ was selected. The relation (5.29) is then satisfied for the profile (5.15) with $q_{c} \approx 0.1$. The amplitude $\epsilon$ was chosen such that the prediction and the measurement are the same at the peak position.

Fiedler \& Mensing (1985) measured the root-mean-square velocity of the fundamental component at $y=0$, normalized by $U_{1}^{*}$. Interestingly, the disturbance amplitude, as displayed in figures 18 and 22 in their paper, experiences more reduction and exhibits more pronounced oscillation than that in the experiment of WW. The measured quantity, denoted here by $A_{\mathrm{exp}}$, is related to $A$ in the present theory by

$$
A_{\mathrm{exp}}=\epsilon\left(\alpha \bar{U}_{c}^{\prime}\right)^{-1} A / \sqrt{2} .
$$

The comparison is shown in figure 10. There is a fairly good agree of quantitative agreement. With non-parallelism being included, the prediction for the initial growth in the linear regime is quite accurate. The theory captures not only the overall nonlinear devel- 
opment, but also mimics the rather detailed feature of non-monotonic attenuation. The measurement is less oscillatory than the theoretical result, which is also the case for the experiment of WW. The reason for this remains unclear. It might be that the closure model for fine-scale turbulence is not sophisticated enough, or that the disturbances in experiments have narrow spectral bands rather than being monochromatic as assumed in calculations. The possibility of the smoothing effect of phase averaging experimental data cannot be ruled either.

In order to examine the sensitivity of the prediction to uncertainty of the parameters. Further calculations were carried out with the value of $\chi$ being doubled, halved (not shown) and set to zero, and also with $q_{c}=0.3$. In all these cases, the evolution retains its overall feature. While the behaviour of the solution is remarkably robust with respect to $\chi$ and $q_{c}$, nonlinearity and non-parallelism are both instrumental. With the former being ignored, the disturbance would feature a too broader peak and decay monotonically as is indicated by the linear solution in the figure. On the other hand, if non-parallelism is neglected, the theory would fail to predict attenuation.

Finally, figure 11 displays contours of the critical-layer vorticity of the CS at four representative streamwise locations. They illustrate the roll-up process leading to formation of Kelvin-Helmholtz rollers.

The different performance of the theory, i.e. a fairly good quantitative agreement with the experiment of Fiedler \& Mensing (1985) versus a merely qualitative agreement with that of WW, requires some comments. For the latter case, we took the parameter $q_{c}$ in the mean-flow profile (5.15) to be 0.9 rather than 0.67 suggested by experiments because latter value led to inconsistency in the neutral frequencies. However, it might be possible that the assumed profile (5.15) is not entirely appropriate thereby causing the inaccuracy of the prediction.

\section{Summary and conclusions}

In this paper, a nonlinear theory was proposed to describe the dynamics of large-scale coherent structures in free shear layers. The formulation was based on decomposing the flow field into a mean flow, coherent fluctuations and small-scale turbulence, but differs from the existing approach of triple decomposition in that the mean-flow induced by CS is treated as part of the organised motion. The time- and phase-averaged Reynolds stresses of fine-scale turbulence, which influence the mean flow and CS respectively, are modelled by eddy-viscosity models, of which the one for the phase-averaged stresses allows for possible time-relaxation effects.

In the limit of large Reynolds numbers, the nonlinear non-equilibrium critical-layer theory for instability modes on laminar flows was adapted to CS on turbulent free shear layers. In addition to accounting for the impact of small-scale fluctuations, non-parallelism associated with the fast spreading of the mean flow was retained along with nonlinear, non-equilibrium and viscous effects, at leading order in the critical layer. This leads to an interesting evolution system consisting of an amplitude equation coupled with a strongly nonlinear vorticity equation. Non-parallelism influences, as is well known, the local growth rate, but was found also to contribute the so-called translating critical-layer effect. The latter remains dormant in the linear regime and becomes active only during the nonlinear stage. The simultaneous interplay with nonlinearity, non-equilibrium and (in principle) viscosity means that non-parallelism operates rather differently from that in the theories

of Crighton \& Gaster (1976) and Goldstein \& Hultgren (1988), where its influence is of higher order.

With appropriately specified initial and boundary conditions, the nonlinear evolution 
system was solved numerically. The solutions were found to capture main characteristics observed of CS during their growth and attenuation phases. The combination of nonlinearity and non-parallelism turned out to be crucial. Owing to its strongly nonlinear nature, the theory was able to predict vortex roll-up, which is the hallmark of CS. Detailed comparisons were made with experiments, and a fair degree of quantitative agreement was achieved for one set of experimental data.

The present work represents just a preliminary step to model nonlinear dynamics of CS and is by no means complete. The closure models proposed for the time- and phaseaveraged Reynolds stresses of small-scale turbulence are rather primitive. Indeed, the model for the time-averaged Reynolds stresses was not implemented as we chose instead the mean-flow profile on an empirical and approximate basis. This creates some uncertainty regarding the profile to be used and also limits the predictive power of the theory. With the time-delay eddy viscosity model for the phase-averaged Reynolds stresses, random fluctuations were found to exert little impact on CS, which appeared to evolve inviscidly. However, it remains to be checked whether the conclusion holds when sophisticated closure models were employed. Possibly, they would account for some of the discrepancies between the measurements and the current prediction.

With the current or the prospective improved models, the present work could be extended to three-dimensional disturbances and to other free shear flows. The latter include plane wakes and jets, where sinuous and varicos modes co-exist (Wygnanski et al. 1986). It would be interesting to investigate their mutual interactions and perform comparisons with available experimental measurements (Marasli et al. 1989, 1991, 1992). The formulation could readily be generalized to circular jets by accounting for the circumferential curvature. Furthermore, once the nonlinear evolution of CS is described, the noise generated by them could be predicted by following the approaches of $\mathrm{Wu}(2005)$ and $\mathrm{Wu} \&$ Huerre (2009). Work along these lines are in progress.

Finally, it is worth pointing out that although inclusion of non-parallelism as a leadingorder effect in the present paper was formalised by assuming a distinguished size of the turbulent Reynolds number, the significance of non-parallelism is not restricted to turbulent shear flows. In the laminar case, it is customarily assumed that the base flow evolves on the scale of $O\left(R \delta^{*}\right)$, that is, $\mathrm{d} \theta^{*} / \mathrm{d} x^{*}=O\left(R^{-1}\right)$. In reality, however, the spreading rate is not solely controlled by viscous diffusion; other factors, such as the state of the oncoming boundary layer and conditions on the splitter plate, may make a significant difference. The spreading rate turns to be much greater than $O\left(R^{-1}\right)$ in general: typically, $\mathrm{d} \theta^{*} / \mathrm{d} x^{*}$ is of $O\left(10^{-2}\right)$ whereas $R^{-1}=O\left(10^{-3}\right)$. It is therefore reasonable to treat $\mathrm{d} \theta^{*} / \mathrm{d} x^{*}$ as an independent quantity. For both laminar and turbulent flows, the relevant parameter measuring the non-parallel-flow effect is $\epsilon^{-1} \mathrm{~d} \theta^{*} / \mathrm{d} x^{*}$ (see (5.18)), which is usually of $O(1)$ for laminar flows too, suggesting that non-parallelism may also need to be included at leading order in the same fashion as it was done in the present paper.

The authors would like to thank Prof. J. T. C. Liu for interesting discussions, and the referees for helpful suggestions and comments. This research is supported NSFC (grant 11472190).

\section{A Detailed derivation of equation (3.45)}

Let $\mathcal{D}_{0} \equiv\left(\frac{\partial}{\partial \tau}+\frac{\partial}{\partial \bar{x}}\right)$ act on both sides of (3.44) with the $O\left(M^{-1}\right)$ term omitted. Then

$$
\left(\Lambda_{0} \bar{x}-\mathrm{i} S_{0} \Lambda_{1}+\Lambda_{1} \frac{\partial}{\partial \tau}+\Lambda_{2} \frac{\partial}{\partial \bar{x}}\right) \mathcal{D}_{0} A+\Lambda_{0} A=\int_{-M}^{M} \mathcal{D}_{0} Q_{1} \mathrm{~d} \eta+O\left(M^{-1}\right)
$$




$$
=-2 M\left(\mathcal{D}_{0}-\mathrm{i} S_{0}-\mathrm{i} \chi_{2} \bar{x}\right) A-\mathrm{i} I_{11}+O\left(M^{-1}\right),
$$

where use has been made of (3.38) and (3.41). The above equation may be written as

$$
\Lambda_{2} \mathcal{D}_{0}^{2} A+\Lambda_{0} A=-\left(\Lambda_{0} \bar{x}-\mathrm{i} S_{0} \Lambda_{1}+\Lambda_{d} \frac{\partial}{\partial \tau}\right) \mathcal{D}_{0} A-2 M\left(\mathcal{D}_{0}-\mathrm{i} S_{0}-\mathrm{i} \chi_{2} \bar{x}\right) A-\mathrm{i} I_{11}+O\left(M^{-1}\right)
$$

where we have put $\Lambda_{d}=\Lambda_{1}-\Lambda_{2}$.

Using (A.2) in (3.44) to eliminate $\mathcal{D}_{0}^{2} A$, we obtain a first-order equation (with respect to $\partial / \partial \bar{x})$,

$$
\begin{aligned}
& \left(\Lambda_{0} \bar{x}-\mathrm{i} S_{0} \Lambda_{1}\right) A+\Lambda_{1} \frac{\partial A}{\partial \tau}+\Lambda_{2} \frac{\partial A}{\partial \bar{x}} \\
& =\frac{4}{\Lambda_{2}}\left(\mathcal{D}_{0}-\mathrm{i} S_{0}-\mathrm{i} \chi_{2} \bar{x}\right) A+\frac{2}{M \Lambda_{2}}\left[\Lambda_{0} A+\left(\Lambda_{0} \bar{x}-\mathrm{i} S_{0} \Lambda_{1}+\Lambda_{d} \frac{\partial}{\partial \tau}\right) \mathcal{D}_{0} A+2 \mathrm{i} I_{11}\right] \\
& \quad+I_{10}+\frac{2 \mathrm{i}\left(S_{0}+\chi_{2} \bar{x}\right)}{M} \mathcal{D}_{0} A+\frac{2 \mathrm{i} \chi_{2}}{M} A+O\left(M^{-2}\right)
\end{aligned}
$$

which may be arranged to

$$
\begin{aligned}
\mathcal{D}_{1} \mathcal{D}_{0} A= & I_{10}-\left(\Lambda_{0} \bar{x}-\mathrm{i} S_{0} \Lambda_{1}+\Lambda_{d} \frac{\partial}{\partial \tau}\right) A-\frac{4 \mathrm{i}\left(S_{0}+\chi_{2} \bar{x}\right)}{\Lambda_{2}} A+\frac{2 \mathrm{i} \chi_{2}}{M} A \\
& +\frac{2 \Lambda_{0}}{M \Lambda_{2}} A+\frac{2 \mathrm{i} I_{11}}{M \Lambda_{2}}+O\left(M^{-2}\right),
\end{aligned}
$$

where we have introduced

$$
\mathcal{D}_{1}=\left[\Lambda_{2}-\frac{2 \mathrm{i}\left(S_{0}+\chi_{2} \bar{x}\right)}{M}-\frac{4}{\Lambda_{2}}-\frac{2}{M \Lambda_{2}}\left(\Lambda_{0} \bar{x}-\mathrm{i} S_{0} \Lambda_{1}+\Lambda_{d} \frac{\partial}{\partial \tau}\right)\right] .
$$

Let $\mathcal{D}_{0}$ act on (A.4). Then

$$
\begin{aligned}
\mathcal{D}_{1} \mathcal{D}_{0}^{2} A= & -2 M\left(\mathcal{D}_{0}-\mathrm{i} S_{0}-\mathrm{i} \chi_{2} \bar{x}\right) A-\mathrm{i} I_{11}+\frac{4 \Lambda_{0}}{M \Lambda_{2}} \mathcal{D}_{0} A-\left(\Lambda_{0}+\frac{4 \mathrm{i} \chi_{2}}{\Lambda_{2}}\right) A \\
& -\left(\Lambda_{0} \bar{x}-\mathrm{i} S_{0} \Lambda_{1}+\Lambda_{d} \frac{\partial}{\partial \tau}\right) \mathcal{D}_{0} A-\frac{4\left(\mathrm{i} S_{0}+\mathrm{i} \chi_{2} \bar{x}\right)}{\Lambda_{2}} \mathcal{D}_{0} A+\frac{4 \mathrm{i} \chi_{2}}{M} \mathcal{D}_{0} A \\
& -\frac{2 \mathrm{i}}{M \Lambda_{2}}\left\{\mathrm{i} I_{12}+\chi \int_{-M}^{M} \eta \frac{\partial}{\partial \eta} Q_{1} \mathrm{~d} \eta+\mathrm{i} \int_{-M}^{M} \eta \frac{\partial}{\partial \eta}\left(A^{*} Q_{2}-A Q_{0}\right) \mathrm{d} \eta+O\left(M^{-1}\right)\right\} \\
= & \left\{-2 M-\left(\Lambda_{0} \bar{x}-\mathrm{i} S_{0} \Lambda_{1}+\Lambda_{d} \frac{\partial}{\partial \tau}\right)-\frac{4 \mathrm{i}\left(S_{0}+\chi_{2} \bar{x}\right)}{\Lambda_{2}}+\frac{4 \Lambda_{0}}{M \Lambda_{2}}+\frac{4 \mathrm{i} \chi_{2}}{M}\right\} \mathcal{D}_{0} A \\
& +\left\{2 \mathrm{i} M\left(S_{0}+\chi_{2} \bar{x}\right)-\left(\Lambda_{0}+\frac{4 \mathrm{i} \chi_{2}}{\Lambda_{2}}\right)\right\} A-\mathrm{i} I_{11} \\
& +\frac{2}{M \Lambda_{2}}\left(I_{12}+\mathrm{i} \chi I_{10}-A^{*} I_{20}\right)+O\left(M^{-2}\right) .
\end{aligned}
$$

In the last step above, integration by parts is performed. Now eliminating the term $\mathcal{D}_{0} A$ between (3.44) and (A.5) and rearranging, we finally obtain (3.45), the coefficients in which are found as

$$
\begin{gathered}
\tilde{\Lambda}_{1}=\left(q_{1}-q_{2} \bar{x}\right)\left[\Lambda_{1}-\frac{2 \mathrm{i}\left(S_{0}+\chi_{2} \bar{x}\right)}{M}\right]+\frac{2}{M}\left[p_{1}+p_{2} \bar{x}-\frac{\Lambda_{d}\left(\Lambda_{0} \bar{x}-\mathrm{i} S_{0} \Lambda_{1}\right)}{\Lambda_{2}}+\frac{2 \mathrm{i} \chi_{2} \Lambda_{d}}{M \Lambda_{2}}\right], \\
\tilde{\Lambda}_{2}=\left(q_{1}-q_{2} \bar{x}\right)\left[\Lambda_{2}-\frac{2 \mathrm{i}\left(S_{0}+\chi_{2} \bar{x}\right)}{M}\right]+\frac{2}{M}\left(p_{1}+p_{2} \bar{x}\right),
\end{gathered}
$$




$$
\begin{gathered}
\tilde{\Lambda}_{d, j}=\frac{2 \Lambda_{d}}{M}\left[1-\frac{2 \mathrm{i}\left(S_{0}+\chi_{2} \bar{x}\right)}{M \Lambda_{2}}+\frac{\Lambda_{j}}{\Lambda_{2}}\right] \quad(j=1,2), \\
\tilde{\Lambda}_{0}=\left(q_{1}-q_{2} \bar{x}\right)\left(\Lambda_{0} \bar{x}-\mathrm{i} S_{0} \Lambda_{1}-\frac{2 \mathrm{i} \chi_{2}}{M}\right)+\frac{2}{M}\left(r_{1}+r_{2} \bar{x}\right),
\end{gathered}
$$

with

$$
\begin{gathered}
q_{1}=\Lambda_{2}-\frac{2 \mathrm{i} S_{0}}{M}-\frac{4}{\Lambda_{2}}+\frac{2 \mathrm{i} S_{0} \Lambda_{1}}{M \Lambda_{2}}, \quad q_{2}=\frac{2 \mathrm{i} \chi_{2}}{M}+\frac{2 \Lambda_{0}}{M \Lambda_{2}}, \\
p_{1}=-2 M+\mathrm{i} S_{0} \Lambda_{1}-\frac{4 \mathrm{i} S_{0}}{\Lambda_{2}}+\frac{4 \Lambda_{0}}{M \Lambda_{2}}+\frac{4 \mathrm{i} \chi_{2}}{M}, \quad p_{2}=-\Lambda_{0}-\frac{4 \mathrm{i} \chi_{2}}{\Lambda_{2}}, \\
r_{1}=2 \mathrm{i} S_{0} M-\Lambda_{0}-\frac{4 \mathrm{i} \chi_{2}}{\Lambda_{2}}, \quad r_{2}=2 \mathrm{i} \chi_{2} M .
\end{gathered}
$$




\section{References}

[1] Alper, A. \& Liu, J.T.C. 1978 On the interactions between large-scale structure and fine-grained turbulence in a free shear layer. Part II: The developmentof of spatial interactions in the mean. Proc. R. Soc. Lond. A 359, 497-523.

[2] Antonia, R.A., Browne, L.W.B., Rajagopalan, S. \& Chambers, A.J. 1983 On the organized motion of a turbulent plane jet. J. Fluid Mech. 134, 49-66.

[3] Antonia, R.A., Chambers, A.J., Britz, D. \& Browne, L.W.B. 1986 Organized structures in a turbulent plane jet: topology and contribution to momentum and heat transport. J. Fluid Mech. 172, 211-229.

[4] Benney, D. J. \& Bergeron, F. 1969 A new class of nonlinear waves in parallel flows. Stud. Appl. Math. 48, 181-204.

[5] Batt, R. G. 1975 Some measurements on the effect of tripping the two-dimensional shear layer. AIAA J. 13, 245-247.

[6] Bechert, D.W. \& Pfizenmaier, E. 1975 On the amplification of broadband jet noise by a pure tone excitation. J. Sound Vib. 43, 581-587.

[7] Bishop, K. A., Ffowcs Williams, J. E. \& Smith, W. 1971 On the noise sources of the unsuppressed high-speed jet. J. Fluid Mech. 50, 21-31.

[8] Brown, G. L. \& Roshko, A. 1974 On density effects and large structure in turbulent mixing layers. J. Fluid Mech. 64, 775-816.

[9] Browand, F.K. \& Troutt, T.R. 1980 A note on spanwise structure in the twodimensional mixing layer. J. Fluid Mech. 97, 771-781.

[10] Cantwell, B.J. 1981 Organized motion in turbulent flow. Ann. Rev. Fluid Mech. 13, 457-515.

[11] Cavalieri, A.V.G., Rodriguez, D., Jordan, P., Colonius, T. \& Gervais, Y. 2013 Wavepackets in the velocity field of turbulent jets. J. Fluid Mech. 730, 559-592.

[12] Churilov, S.M. \& Shukhman, I. G. 1994 Nonlinear spatial evolution of helical disturbances to an axial jet. J. Fluid Mech. 281, 371-402.

[13] Cohen, J., Marasli B. \& Levinski, V. 1994 The interaction between the mean flow and coherent structures in turbulent mixing layers. J. Fluid Mech. 260, 81-94.

[14] Cowley, S.J. 1985 Pulsatile flow through distorted channels: low-Strouhal-number and translating critical-layer effect. Q. J. Mech. App. Maths. 38(4) 589-619.

[15] Cowley, S.J. \& Wu, X. 1994 Asymptotic approaches to transition modelling. In Progress in Transition Modelling, AGARD Report 793, Chapter 3, 1-38.

[16] Crighton, D. G. \& Gaster, M. 1976 Stability of slowly diverging jet flow. J. Fluid Mech. 77, 397-413.

[17] Crow, S.C. \& Champagne. F.H. 1971 Orderly structure in jet turbulence. J. Fluid Mech. 48, 547-591.

[18] Dziomba, B. \& Fiedler, H .E. 1985 Effect of initial conditions on two-dimensional free shear layers. J. Fluid Mech. 152, 419-442.

[19] Dimotakis, P.E. \& Brown, G L. 1976 The mixing layer at high Reynolds number: large-structure dynamics and entrainment. J. Fluid Mech. 78, 535-560. 
[20] Estevadeordal, J. \& Kleis, S.J. 2002 Influence of vortex-pairing location on the threedimensional evolution of plane mixing layers. J. Fluid Mech. 462, 43-77.

[21] Fiedler, H. E. \& Mensing, P. 1985 The plane turbulent shear layer with periodic excitation. J. Fluid Mech. 150, 281-309.

[22] Gaster, M., Kit, E. \& Wygnanski, I. 1985 Large-scale structures in a forced turbulent mixing layer. J. Fluid Mech. 150, 23-39.

[23] Goldstein, M.E. 1995 The role of nonlinear critical layers in boundary-layer transition. Phil. Trans. R. Soc. Lond. A 352: 425-442.

[24] Goldstein, M.E. \& Choi, S.-W. 1989 Nonlinear evolution of interacting oblique waves on two-dimensional shear layers. J. Fluid Mech. 207, 97-120.

[25] Goldstein, M.E. \& Choi, S.-W. 1989 Nonlinear evolution of interacting oblique waves on two-dimensional shear layers. J. Fluid Mech. 207, 97-120.

[26] Goldstein, M.E. \& Hultgren, L.S. 1988 Nonlinear spatial evolution of an externally excited instability wave in a free shear layer. J. Fluid Mech. 197, 295-330.

[27] Goldstein, M.E.\& Leib, S.J. 1988 Nonlinear roll-up of externally excited shear layers. J. Fluid Mech. 191, 481.

[28] Gudmundsson, K. \& Colonius, T. 2011 Instability wave models for the near-field fluctuations of turbulent jets. J. Fluid Mech. 689, 97-128.

[29] Haberman, R. 1972 Critical layers in parallel shear flows. Stud. Appl. Maths. 51, 139-161.

[30] Haynes, P.H. \& Cowley, S.J. 1986 The evolution of an unsteady translating nonlinear Rossby-wave critical layer. Geophys. Astrophys. Fluid Dynamics 35, 1-55.

[31] Ho, C.M. \& Huerre, P. 1990 Perturbed free shear layers. Ann. Rev. Fluid. Mech. 16, $365-422$.

[32] Hussain, A. K. M. F. 1983 Coherent structures - reality and myth. Phys. Fluids 26, $2816-2850$.

[33] Hussain, A.F.M.F. \& Clark, A. R. 1981 On the coherent structure of the axisymmetric mixing layer: a flow-visualization study. J. Fluid Mech. 104, 263-294.

[34] Hussain, A.F.M.F.\& Hasan, M.A.Z. 1985 Turbulence suppression in free turbulent shear flows under controlled excitation. Part 2. Jet-noise reduction. J. Fluid Mech. 105, 159-168.

[35] Hussain, A.F.M.F. \& Reynolds, W.C. 1970 The mechanics of an organized wave in turbulent shear flow. J. Fluid Mech. 41, 241-258.

[36] Hussain, A.F.M.F. \& Thompson, C.A. 1980 Controlled symmetric perturbation of the plane jet: an experimental study in the initial region. J. Fluid Mech. 100, 397-431.

[37] Hussain, A.F.M.F. \& Zaman, K.B.M. 1981 The preferred mode of the axisymmetric jet. J. Fluid Mech. 110, 39-71.

[38] Hussain, A.F.M.F. \& Zaman, K.B.M. 1985 An experimental study of organized motions in the turbulent plane mixing layer. J. Fluid Mech. 159, 85-104.

[39] Jordan, P. \& Colonius, T. 2013 Wave packets and turbulent jet noise. Ann. Rev. Fluid Mech. 45, 173-195. 
[40] Kitsios, V., Cordier, L., Bonnet, J. -P., Ooi, A. \& Soria, D J. 2010 Development of a nonlinear eddy-viscosity closure for the triple-decomposition stability analysis of a turbulent channel. J. Fluid Mech. 664, 74-107.

[41] Liu, J.T.C. \& Merkine, L. 1976 On the interactions between large-scale structure and fine-grained turbulence in a free shear flow. Proc. R. Soc. Lond. A. 352, 213-247.

[42] Liu, J.T.C. 1989 Cohenrent structures in transitional and turbulent free shear flows. Ann. Rev. Fluid Mech. 21, 285-315.

[43] Oberleithner, K., Rukes, L. \& Soria, J. 2014 Mean flow stability analysis of oscillating jet experiments. J. Fluid Mech. 757, 1-32.

[44] Mankbadi, R. \& Liu, J.T.C. 1981 A study of large-scale coherent structures and fine-grained turbulence in a round jet. Phil. Trans. R. Soc. Lond. A. 298, 541-602.

[45] Marasli, B., Champagne, F.H. \& Wygnanski, I. 1989 Modal decomposition of velocity signals in a plane, turbulent wake. J. Fluid Mech. 198, 255-273.

[46] Marasli, B., Champagne, F.H. \& Wygnanski, I. 1991 On linear evolution of unstable disturbances in a plane turbulent wake. Phys. Fluids A3(4), 665-674.

[47] Marasli, B., Champagne, F.H. \& Wygnanski, I. 1992 Effect of travelling waves on the growth of a plane turbulent wake. J. Fluid Mech. 235, 511-528.

[48] Meyer, T.R., Dutton, J.C. \& Lucht, R.P. 2006 Coherent structures and turbulent molecular mixing in gaseous planar shear layers. J. Fluid Mech. 558, 179-205.

[49] Nygaard, K. \& Glezer, A. 1994 The effect of phase variations and cross-shear on vortical structures in a plane mixing layer. J. Fluid Mech. 276, 21-59.

[50] Oster, D. \& Wygnanski, I. 1982 The forced mixing layer between parallel streams. J. Fluid Mech. 123, 91-130.

[51] Redekopp, L.G. 1977 On the theory of solitary Rossby wave. J. Fluid Mech. 82, 725-745.

[52] Reynolds, W.C. \& Hussain, A.K.M.F. 1972 The mechaniscs of an organised wave in turbulent shear flow. Part 3. Theoretical models and comparisons with experiments. J. Fluid Mech. 54, 263-288.

[53] Roshko, A. 1976 Structure of turbulent shear flows: a new look. AIAA J. 14 (10), 1349-1357.

[54] Suzuki, T. 2013 Coherent noise sources of a subsonic round jet investigated using hydrodynamic and acoustic phased-microphone arrays. J. Fluid Mech. 730, 659-698.

[55] Suzuki, T. \& Colonius, T. 2006 Instability waves in a subsonic round jet detected using a near-field phased microphone array. J. Fluid Mech. 565, 197-226.

[56] Tam, C. K. W. \& Burton, D. E. 1984 Sound generated by instability waves of supersonic flow. Part 2. Axisymmetric jets. J. Fluid Mech. 138, 273-295.

[57] Weisbrot, I., Einav, S. \& Wygnanski, I. 1982 The nonunique rate of spread of the two dimensional mixing layer. Phys. Fluids 25, 1691-1693.

[58] Weisbrot, I. \& Wygnanski, I. 1988 On coherent structures in a highly excited mixing layer. J. Fluid Mech. 195, 137-159.

[59] Winant, C.D. \& Browand, F.K. 1974 Vortex pairing: the mechanism of turbulent mixing-layer growth at moderate Reynolds number. J. Fluid Mech. 63, 237-255. 
[60] Wu, X. 2005 Mach wave radiation of nonlinearly evolving supersonic instability modes in shear layers. J. Fluid Mech. 523, 121-159.

[61] Wu, X. \& Huerre, P. 2009 Low-frequency sound radiated by a nonlinear modulated wavepacket of helical modes on a subsonic circular jet. J. Fuild Mech. 637, 173-211.

[62] Wu, X., Lee, S.S. \& Cowley, S.J. 1993 On the weakly nonlinear instability of shear flows to pairs of oblique waves: the Stokes layer as a paradigm. J. Fluid Mech. 253, 681-720.

[63] Wu, X. \& Tian, F. 2012 Spectral broadening and flow randomization in free shear layers. J. Fluid Mech. 706, 431469.

[64] Wu, X. \& Zhou, H. 1989 Linear instability of turbulent boundary layer as a mechanism for the generation of large scale coherent structures. Chinese Science Bulletin (English Edition) 34 (20), 1685-1688.

[65] Wygnanski, I., Champagne, F.H. \& Marasli, B. 1986 On the large-scale structures in two-dimensional, small-deficit, turbulent wakes. J. Fluid Mech. 168, 31-71.

[66] Wygnanski, I., Oster, D., Fiedler, H. \& Dziomba, B. 1979 On the perseverance of a quasi-two-dimensional eddy-structure in a turbulent mixing layer. J. Fluid Mech. 93, 325-335.

[67] Wygnanski, I. \& Petersen, R. A. 1987 Coherent motion in excited free shear flows. AIAA J. 25, 201-213.

[68] Wygnanski, I. \& Weisbrot, I. 1988 On the pairing process in an excited plane turbulent mixing layer. J. Fluid Mech. 195, 161-173.

[69] Zaman, K.B.M. \& Hussain, A.F.M.F. 1980 Vortex pairing in a circular jet under controlled excitation. Part 1. General jet response. J. Fluid Mech. 101, 449-491. 